CPT-96/P.3408

November 1996

(revised May 1997)

\title{
Electroweak Effective Charges and their relation to Physical Cross Sections
}

\author{
J. Papavassiliou用, E. de Rafael and N.J. Watson
}

Centre de Physique Théorique, CNRS-Luminy, Case 907, F-13288 Marseille Cedex 9, France.

\begin{abstract}
In quantum electrodynamics with fermions $f=e, \mu \ldots$, knowledge of the vacuum polarization spectral function determined from the tree level $e^{+} e^{-} \rightarrow f^{+} f^{-}$cross sections, together with a single low energy measurement of the fine structure constant $\alpha$, enables the construction of the one-loop effective charge $\alpha_{\text {eff }}\left(q^{2}\right)$ for all $q^{2}$. It is shown how an identical procedure can be followed in the electroweak sector of the Standard Model to construct three gauge-, scale- and scheme-independent one-loop electroweak effective charges and an effective weak mixing angle from the tree level $e^{+} e^{-} \rightarrow W^{+} W^{-}, Z H$ and $e^{+} \nu_{e} \rightarrow W^{+} Z, W^{+} \gamma, W^{+} H$ differential cross sections, together with three low energy measurements, which may be chosen to be $\alpha$ and the masses of the $W$ and $Z$ bosons. It is found that the corresponding proper self-energy-like functions thus constructed are identical to those obtained in the pinch technique framework. In this way, it is shown how the concept of effective charges in the electroweak Standard Model is as well-defined and unique as in quantum electrodynamics.
\end{abstract}

${ }^{*}$ Present address: Dept. of Physics and Astronomy, University of Manchester, Manchester M13 9PL, UK.

${ }^{\dagger}$ Present address: Institut de Physique Nucléaire, Université de Paris-Sud, F-91406 Orsay Cedex, France. 


\section{Introduction}

The possibility of extending the concept of an effective charge [1] from quantum electrodynamics to non-abelian gauge theories is of fundamental interest for at least three reasons. First, in quantum chromodynamics, the existence of an effective charge analogous to that of QED is explicitly assumed in renormalon analyses of the behaviour of pertubation series at high orders [2]. The ability to identify directly and unambiguously the infinite subset of gluon self-energy-like radiative corrections that one is summing in such analyses is important in order to provide a well-defined basis for renormalon calculus. Second, in theories involving unstable particles, e.g. the Standard Model, the presence of such particles necessarily requires the Dyson summation of infinite subsets of radiative corrections in order to regulate the singularities which otherwise occur in the corresponding tree level propagators [3]. Such summations, directly linked to the concept of an effective charge, are essential for the evaluation of physical amplitudes at arbitrary values of the kinematic parameters. Third, in theories involving disparate energy scales, e.g. grand unified theories, the extraction of accurate lowenergy predictions requires an exact treatment of threshold effects due to heavy particles [4]. The ability to construct a set of effective charges, valid for all momenta $q^{2}$ and not just the asymptotic regime governed by the renormalization group $\beta$-functions, would automatically provide the natural way to account for such threshold effects. In all cases, the fundamental problem is the gauge dependence, and hence ambiguity, of the gauge boson self-energies in a non-abelian gauge theory. This gauge dependence necessarily means that the gauge boson self-energies in such theories are not directly related to measurable quantities.

Recently however, there has been substantial progress in understanding how the QED concept of an effective charge can be extended to non-abelian gauge theories [5 8]. The theoretical framework which has enabled this progress is the pinch technique (PT) [9]. The PT is a well-defined algorithm for the rearrangement of conventional gauge-dependent one-loop $n$-point functions to construct individually gauge-independent one-loop "effective" $n$-point functions. This rearrangement of perturbation theory is based on the systematic use of the tree level Ward identities of the theory to cancel in Feynman amplitudes all factors of longitudinal four-momentum associated with gauge fields propagating in loops. In addition to being gauge-independent, the PT "effective" $n$-point functions display many desirable theoretical properties. In particular, they obey the same Ward identities as the corresponding tree level functions.

The purpose of this paper is to discuss the relationship between effective charges and the cross sections for certain physical processes in the electroweak sector of the Standard Model. It is well known that in QED with fermions $f=e, \mu \ldots$, the imaginary part of, e.g., the muon contribution to the one-loop vacuum polarization is directly related to the tree level cross section for the process $e^{+} e^{-} \rightarrow \mu^{+} \mu^{-}$. This relation is a result purely of the unitarity of the S-matrix $S=I+i T$, expressed in the optical theorem for the particular case of 


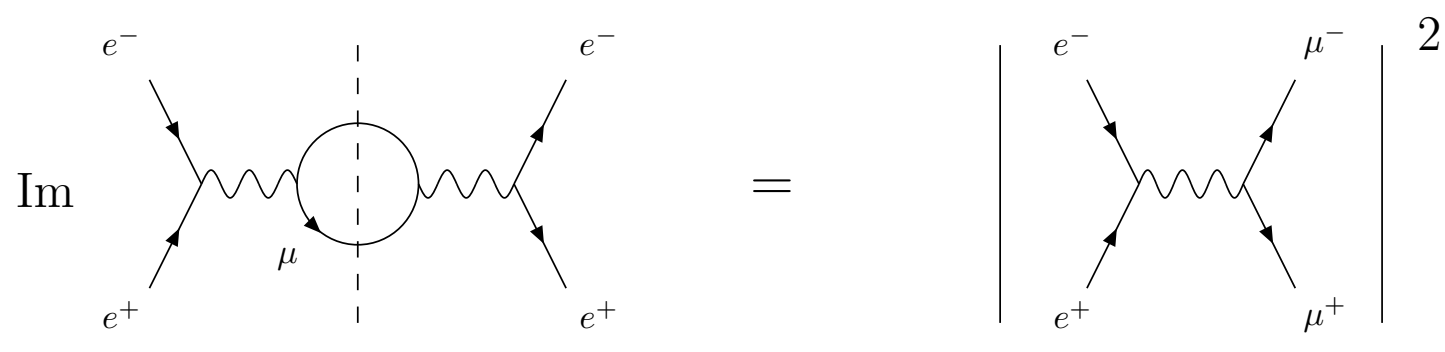

Fig. 1. The relation between the imaginary part of the muon contribution to the one-loop vacuum polarization and the tree level cross section $\sigma\left(e^{+} e^{-} \rightarrow \mu^{+} \mu^{-}\right)$in QED.

forward scattering in the process $e^{+} e^{-} \rightarrow e^{+} e^{-}$:

$$
\operatorname{Im}\left\langle e^{+} e^{-}|T| e^{+} e^{-}\right\rangle=\frac{1}{2} \sum_{i} \int d \Gamma_{i}\left|\left\langle e^{+} e^{-}|T| i\right\rangle\right|^{2} .
$$

In Eq. (1.1), the sum on the right hand side is over all on-shell physical states $|i\rangle$ compatible with the quantum numbers of $\left|e^{+} e^{-}\right\rangle$; in each case the integral is over the available phase space $\Gamma_{i}$. The tree level contribution of the muon pair $\left|\mu^{+} \mu^{-}\right\rangle$to the r.h.s. of Eq. (1.1) and the corresponding imaginary part of the muon contribution to the one-loop vacuum polarization on the 1.h.s. of Eq. (1.1) are illustrated schematically in Fig. 1. The muon contribution to the renormalized one-loop vacuum polarization may thus be reconstructed directly from the tree level cross section $\sigma\left(e^{+} e^{-} \rightarrow \mu^{+} \mu^{-}\right)$via a once-subtracted dispersion relation. Similarly, the tree level contributions of the electron-positron pair $\left|e^{+} e^{-}\right\rangle$to the r.h.s. of Eq. (1.1) and the corresponding imaginary parts of the electron contribution to the one-loop diagrams on the 1.h.s. of Eq. (1.1) are illustrated schematically in Fig. 2. As indicated explicitly in Fig. 2, the imaginary part of the one-loop self-energy [box] diagram on the l.h.s. corresponds to the direct contribution of the $s$-channel [ $t$-channel] tree level photon exchange diagram on the r.h.s., while the imaginary part of the one-loop vertex diagram on the l.h.s. corresponds to the interference contribution of the tree level diagrams on the r.h.s. The electron contribution to the vacuum polarization may thus be reconstructed directly from the self-energy-like componentf of the tree level Bhahba scattering cross section $\sigma\left(e^{+} e^{-} \rightarrow e^{+} e^{-}\right)$again via a once-subtracted dispersion relation. Knowledge of each such contribution to the vacuum polarization, together with a single low energy measurement of the fine structure constant $\alpha=1 / 137.036 \ldots$, then enables the QED one-loop effective charge $\alpha_{\text {eff }}\left(q^{2}\right)$ to be uniquely constructed for all values of its argument.

\footnotetext{
$\ddagger$ The self-energy-like component of the experimental Bhabha cross section may be projected out from the full cross section using a procedure analogous to that to be described here in Sec. 8 .
} 

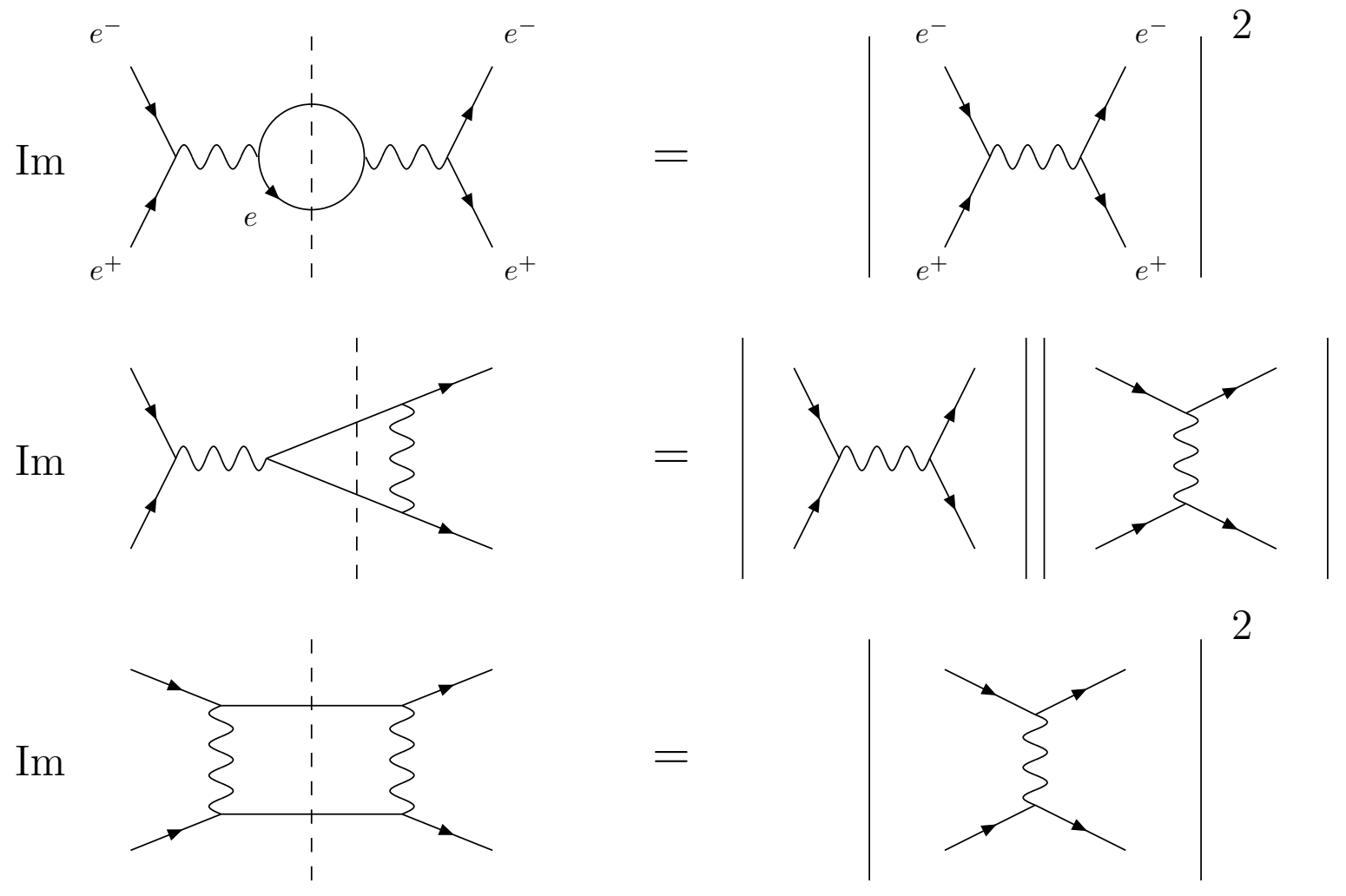

Fig. 2. The relation between the imaginary parts of the electron contribution to the one-loop vacuum polarization, vertex and box diagrams and the components of the tree level cross section $\sigma\left(e^{+} e^{-} \rightarrow e^{+} e^{-}\right)$in QED.

When QED is embedded in the electroweak sector of the Standard Model, there occur further lowest order contributions to the relation Eq. (1.1). The tree level contributions of the gauge boson pair $\left|W^{+} W^{-}\right\rangle$to the r.h.s. of Eq. (1.1) and the corresponding imaginary parts of the contributions of the $W$ and its associated would-be Goldstone boson and ghost to the one-loop diagrams for $e^{+} e^{-} \rightarrow e^{+} e^{-}$on the 1.h.s. of Eq. (1.1) are illustrated schematically in Fig. 3 (for simplicity, the coupling of the Higgs to $e^{+} e^{-}$has been neglected). There are two basic and well-known observations to make regarding the equation illustrated in Fig. 3. First, on the r.h.s. of Fig. 3, each of the contributions corresponding to the individual tree level diagrams violates unitarity [16]. It is only when the contributions, direct plus interference, from all three diagrams are combined that, as a result of extensive cancellations, the overall contribution on the r.h.s. of Fig. 3 is well-behaved at high energies. Second, on the 1.h.s. of Fig. 3, each of the contributions due to the imaginary parts of the one-loop self-energy, vertex and box diagrams is individually gauge-dependent [17]. It is only when the contributions are combined that the gauge dependencies, in particular the unphysical thresholds that in general 


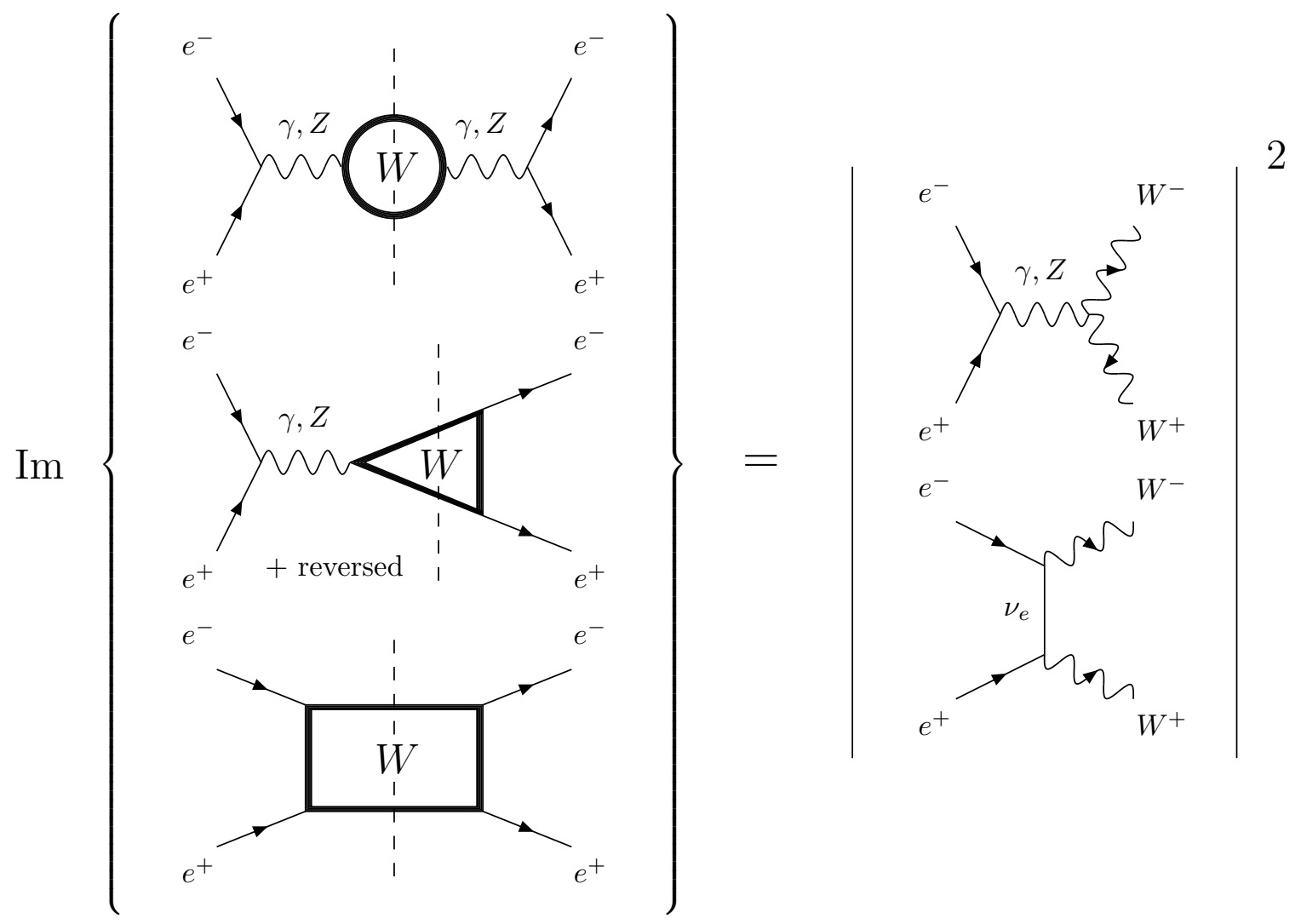

Fig. 3. The schematic representation of the tree level contribution of the gauge boson pair $\left|W^{+} W^{-}\right\rangle$to the r.h.s. of Eq. (1.1) and the corresponding imaginary parts of the contributions of the $W$ and its associated would-be Goldstone boson and ghost, collectively denoted by " $W$ ", to the one-loop self-energy, vertex and box diagrams for the process $e^{+} e^{-} \rightarrow e^{+} e^{-}$on the 1.h.s. of Eq. (1.1) in the electroweak Standard Model.

occur, cancel and the overall contribution on the l.h.s. of Fig. 3 is gauge-independent. Either of these facts alone is sufficient to prevent the unitarity relation illustrated in Fig. 3 from holding for the individual diagrammatic contributions which occur on each side. Thus, the simple QED relation between the components of the tree level cross sections for the interaction of on-shell particles and the imaginary parts of the corresponding one-loop self-energy, vertex and box diagrams is apparently lost.

In this paper, it is shown how the QED-like correspondence between individual contributions on each side of Eq. (1.1) may be maintained in the electroweak Standard Model. The starting point is a simple re-analysis of the calculation of the tree level cross section for the process $e^{+} e^{-} \rightarrow W^{+} W^{-}$. The crucial observation is that the cancellation mechanism responsible for the good high energy behaviour of the overall cross section occurs directly at the level of the tree level Feynman diagrams. Implementing this cancellation at the very first 
step in the calculation, rather than the very last, the cross section is shown to decompose naturally into components which are individually well-behaved at high energy. Then, by direct analogy with QED, the resulting self-energy-like cross section components may be used to define manifestly gauge-independent $W^{+} W^{-}$contributions to photon and $Z$ one-loop renormalized self-energies via dispersion relations. These self-energy contributions are found to be identical to those obtained in the pinch technique. It is then shown how these self-energies, together with that for the $W$ gauge boson, define three gauge-independent electroweak effective charges and an effective weak mixing angle. These four effective quantities are shown to be renormalization scale- and scheme-independent, and at high energies to match on to the corresponding running quantities defined from the two electroweak $\beta$-functions. Finally, it is described how the self-energy-like components of the $e^{+} e^{-} \rightarrow W^{+} W^{-}$cross section used to construct the $W^{+} W^{-}$contributions to the photon and $Z$ self-energies may be extracted directly from experiment. Explicit expressions are given for the required projection functions in the simplified case in which the weak mixing angle is set to zero. In this way, it is shown how the concept of an effective charge in the electroweak Standard Model is as well-defined and unique as in QED.

The paper is organized as follows. In Sec. 2, a brief review is given of properties of the vacuum polarization and effective charge in QED. In Sec. 3, the re-analysis of the calculation of the tree level cross section for $e^{+} e^{-} \rightarrow W^{+} W^{-}$is presented, together with that for $e^{+} e^{-} \rightarrow$ $Z H$, where $H$ is the Higgs boson. In Sec. 4, the definitions are given of the $W$ and $Z H$ contributions to photon and $Z$ one-loop self-energies in terms of the cross section components. In Sec. 5, the corresponding set of effective charges and the effective weak mixing angle are constructed. In Sec. 6, it is shown how these four effective quantities are related to the two running couplings defined from the renormalization group. In Sec. 7, a comparison is made between the effective charges constructed here and those obtained in the background field method. In Sec. 8, the extraction of the required cross section components from experiment is described. The paper finishes with our conclusions in Sec. 9. Some technical details are relegated to three Appendices. Throughout, we consider only gauge field contributions to one-loop self-energies, since fermion and scalar contributions are standard. 3

\section{The Effective Charge in QED}

We first review some basic properties of the vacuum polarization and effective charge in QED with fermions $f=e, \mu \ldots$. For more details see, e.g., Refs. [19].

i) The vacuum polarization function $\Pi\left(q^{2}\right)$ is gauge-independent at all $q^{2}$ and to all orders in perturbation theory.

ii) At the one-loop level, the imaginary part of $\Pi\left(q^{2}\right)$ is directly related, via the optical theorem, to the tree level cross sections for the physical processes $e^{+} e^{-} \rightarrow f^{+} f^{-}$. The

\footnotetext{
${ }^{\S}$ For a recent extensive discussion of fermion loop effects in $e^{+} e^{-}$annihilation, see Ref. [18].
} 
differential cross section for these processes is given by

$$
\begin{aligned}
& \frac{d \sigma\left(e^{+} e^{-} \rightarrow f^{+} f^{-}\right)}{d \Omega}=\frac{\alpha^{2}}{4 s}\left\{\frac{\beta_{f}}{\beta_{e}}\left[3-\beta_{e}^{2}-\beta_{f}^{2}+\beta_{e}^{2} \beta_{f}^{2} \cos ^{2} \theta\right]\right. \\
& +\delta_{e f} \frac{1}{(1-\cos \theta)} \frac{1}{\beta_{e}^{2}}\left[-6+4 \beta_{e}^{2}-4 \beta_{e}^{2}\left(2-\beta_{e}^{2}\right) \cos \theta-2 \beta_{e}^{4} \cos ^{2} \theta\right] \\
& \left.\quad+\delta_{e f} \frac{1}{(1-\cos \theta)^{2}} \frac{1}{\beta_{e}^{4}}\left[4+6 \beta_{e}^{2}+4 \beta_{e}^{2}\left(2-\beta_{e}^{2}\right) \cos \theta+2 \beta_{e}^{4} \cos ^{2} \theta\right]\right\} \vartheta\left(s-4 m_{f}^{2}\right),
\end{aligned}
$$

where $\alpha=e^{2} / 4 \pi, s$ is the square of the total centre of mass energy, $\theta$ is the centre of mass scattering angle and $\beta_{f}=\sqrt{1-4 m_{f}^{2} / s}$, where $m_{f}$ is the mass of the fermion $f$. In Eq. (2.1), the terms grouped in the first, second and third sets of square parentheses correspond to the self-energy-, vertex- and box-like contributions, respectively, shown in Fig. 2. The fact that the vertex - and box-like contributions only occur in the case $f=e$, i.e. Bhabha scattering, is indicated by the Kronecker deltas $\delta_{e f}$. From the relation Eq. (11.1), the imaginary part of the one-loop contribution of the virtual fermion $f$ to $\Pi\left(q^{2}\right)$ is then given directly by the self-energy-like component of the tree level cross section $\sigma\left(e^{+} e^{-} \rightarrow f^{+} f^{-}\right)$:

$$
\begin{aligned}
e^{2} \frac{1}{s} \operatorname{Im} \Pi^{(f f)}(s) & =\frac{2 \beta_{e}}{\left(3-\beta_{e}^{2}\right)} \sigma_{\text {s.e.l. }}\left(e^{+} e^{-} \rightarrow f^{+} f^{-}\right) \\
& =2 \pi \alpha^{2} \frac{1}{s} \beta_{f}\left\{\frac{2}{3}+\frac{4 m_{f}^{2}}{3 s}\right\} \vartheta\left(s-4 m_{f}^{2}\right),
\end{aligned}
$$

where the subscript "s.e.l." denotes "self-energy-like".

iii) Given a particular contribution to the spectral function $\operatorname{Im} \Pi(s)$, the corresponding contribution to the renormalized vacuum polarization function $\Pi_{R}\left(q^{2}\right)$ can be reconstructed via a once-subtracted dispersion relation. For example, for the one-loop contribution of the fermion $f$, choosing the on-shell renormalization scheme,

$$
\begin{aligned}
\Pi_{R}^{(f f)}\left(q^{2}\right) & =q^{2} \int_{4 m_{f}^{2}}^{\infty} d s \frac{1}{s\left(s-q^{2}\right)} \frac{1}{\pi} \operatorname{Im} \Pi^{(f f)}(s) \\
& =-\frac{\alpha}{\pi} \times\left\{\begin{array}{lc}
-\frac{1}{15} \frac{q^{2}}{m_{f}^{2}}+\mathcal{O}\left(\frac{q^{4}}{m_{f}^{4}}\right) & q^{2} / m_{f}^{2} \rightarrow 0 \\
+\frac{2}{3} \frac{1}{2} \ln \left(-\frac{q^{2}}{m_{f}^{2}}\right)-\frac{5}{9}+\mathcal{O}\left(\frac{m_{f}^{2}}{q^{2}}\right) & -q^{2} / m_{f}^{2} \rightarrow \infty,
\end{array}\right.
\end{aligned}
$$

The coefficient $+2 / 3$ of the logarithmic term in Eq. (2.5), originating from the $+2 / 3$ in the parentheses in Eq. (2.3), is precisely the contribution of the fermion to the first coefficient $\beta_{1}$ of the QED $\beta$-function.

iv) The infinite subset of radiative corrections summed in the Dyson series generated by the one-particle-irreducible vacuum polarization $\Pi_{R}\left(q^{2}\right)$ defines an effective charge which is gauge-, scale-, and scheme-independent to all orders in perturbation theory:

$$
\alpha_{\mathrm{eff}}\left(q^{2}\right)=\frac{e_{R}^{2}}{4 \pi} \frac{1}{1+\Pi_{R}\left(q^{2}\right)}=\frac{e^{2}}{4 \pi} \frac{1}{1+\Pi\left(q^{2}\right)},
$$


where we have used $e_{R}^{2}=\left(Z_{2}^{2} Z_{3} / Z_{1}^{2}\right) e^{2}$ and $1+\Pi_{R}=Z_{3}(1+\Pi)$ together with the QED Ward identity $Z_{1}=Z_{2}$ to write $\alpha_{\text {eff }}\left(q^{2}\right)$ purely in terms of bare quantities.

v) At $-q^{2} / m_{f}^{2} \rightarrow \infty$, the effective charge $\alpha_{\text {eff }}\left(q^{2}\right)$ matches on to the running coupling $\bar{\alpha}\left(q^{2}\right)$ defined from the renormalization group: at the one-loop level,

$$
\lim _{-q^{2} / m_{f}^{2} \rightarrow \infty} \alpha_{\mathrm{eff}}\left(q^{2}\right)=\bar{\alpha}\left(q^{2}\right)=\frac{\alpha_{R}}{1-\frac{\alpha_{R}}{\pi} \beta_{1} \frac{1}{2} \log \left(-q^{2} / m_{f}^{2}\right)},
$$

where $\beta_{1}=+\frac{2}{3} n_{f}$ for $n_{f}$ species of fermion.

vi) At $q^{2}=0, \Pi(0)$ specifies fully the one-loop radiative corrections to the tree level Compton scattering process $\gamma e^{-} \rightarrow \gamma e^{-}$in the limit of vanishing photon energy. The fine structure constant $\alpha=1 / 137.036 \ldots$ which governs Compton scattering in the classical Thomson limit of vanishing photon energy then provides the low-energy definition of the coupling constant for the quantum theory: at the one-loop level,

$$
\alpha=\frac{e^{2}}{4 \pi}(1-\Pi(0))=\frac{e_{R}^{2}}{4 \pi}\left(1-\Pi_{R}(0)+\mathcal{O}\left(e^{4}\right)\right) .
$$

In the on-shell scheme, $\Pi_{R}(0)=0$ and $e_{R}$ is identified with the physical electron charge. At $q^{2}=0$, the effective charge therefore matches on to the fine structure constant: $\alpha_{\text {eff }}(0)=\alpha$.

vii) For $f \neq e$, the imaginary part of the one-loop contribution of the fermion $f$ to the spectral function $\operatorname{Im} \Pi(s)$ is measured directly in the the tree level cross section for $e^{+} e^{-} \rightarrow$ $f^{+} f^{-}$. For $f=e$, it is necessary to isolate the self-energy-like component of the tree level Bhabha cross section. As a result of the form of the numerators in the second and third square parentheses in Eq. (2.1), the decomposition of the Bhabha differential cross section into self-energy-, vertex- and box-like components does not correspond to some set of simple kinematic criteria. The three components of the differential cross section Eq. (2.1) are however linearly independent functions of $\cos \theta$. They may therefore each be projected out from the measured cross section by convolution with appropriately chosen polynomials in $\cos \theta$.

Thus, in QED, knowledge of the spectral function $\operatorname{Im} \Pi(s)$, determined from the tree level $e^{+} e^{-} \rightarrow f^{+} f^{-}$cross sections, together with a single low energy measurement of the fine structure constant $\alpha$ (obtained e.g. from the ac Josephson effect and the quantized Hall effect, or from the anomalous magnetic moment of the electron [20]), enables the construction of the one-loop effective charge $\alpha_{\text {eff }}\left(q^{2}\right)$ for all $q^{2}$. In the remainder of this paper, it will be shown how an identical procedure can be followed in the electroweak sector of the Standard Model to construct a set of gauge-, scale-- and scheme-independent electroweak effective charges.

\section{The Tree Level Processes $e^{+} e^{-} \rightarrow W^{+} W^{-}$and $e^{+} e^{-} \rightarrow Z H$}

In the electroweak sector of the Standard Model, the on-shell states involving the electroweak gauge bosons $W$ and $Z$ which contribute to the r.h.s. of Eq. (1.1) at lowest order are $\left|W^{+} W^{-}\right\rangle$ and $|Z H\rangle$, corresponding to the tree level physical processes $e^{+} e^{-} \rightarrow W^{+} W^{-}$and $e^{+} e^{-} \rightarrow$ $Z H$, where $H$ is the Higgs boson. We shall discuss these processes separately. 


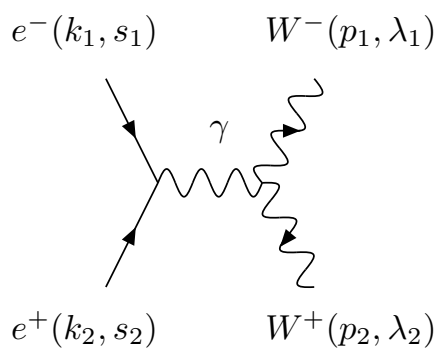

(a)

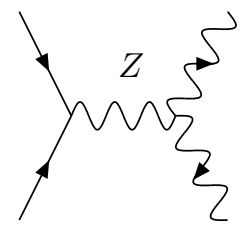

(b)

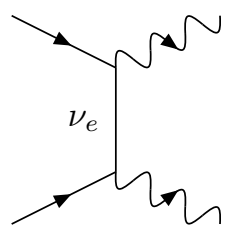

(c)

Fig. 4. The three diagrams which contribute to the process $e^{+} e^{-} \rightarrow W^{+} W^{-}$at tree level for the case of massless fermions.

\section{$3.1 \quad e^{+} e^{-} \rightarrow W^{+} W^{-}$}

We first consider the process $e^{-}\left(k_{1}, s_{1}\right) e^{+}\left(k_{2}, s_{2}\right) \rightarrow W^{-}\left(p_{1}, \lambda_{1}\right) W^{+}\left(p_{2}, \lambda_{2}\right)$, where $s_{1}, s_{2}$ and $\lambda_{1}, \lambda_{2}$ label the polarizations of the on-shell initial and final states. For simplicity, we neglect the fermion mass $\left(m_{e}=0\right)$, and hence the contribution to this process due to Higgs exchange. The three diagrams which contribute at tree level are then as shown in Fig. 4. The relevant kinematic variables are

$$
\begin{aligned}
& s=\left(k_{1}+k_{2}\right)^{2}=\left(p_{1}+p_{2}\right)^{2} \\
& t=\left(k_{1}-p_{1}\right)^{2}=\left(p_{2}-k_{2}\right)^{2}=-\frac{1}{4} s\left(1+\beta^{2}-2 \beta \cos \theta\right),
\end{aligned}
$$

where

$$
\beta=\sqrt{1-\frac{4 M_{W}^{2}}{s}}
$$

and $\theta$ is the centre of mass scattering angle.

The S-matrix element for this process is given by

$$
i\left\langle W^{+} W^{-}|T| e^{+} e^{-}\right\rangle=i \epsilon_{\mu}^{*}\left(p_{1}, \lambda_{1}\right) \epsilon_{\nu}^{*}\left(p_{2}, \lambda_{2}\right) \bar{v}\left(k_{2}, s_{2}\right) T^{\mu \nu} u\left(k_{1}, s_{1}\right),
$$

where $\epsilon_{\mu}^{*}, \epsilon_{\nu}^{*}$ are the $W^{-}, W^{+}$gauge boson polarization vectors, respectively, $u$ and $\bar{v}$ are the fermion spinors and the amputated Green's function $T^{\mu \nu}$ is given by the sum of the diagrams in Fig. 4. The square of the modulus of $\left\langle W^{+} W^{-}|T| e^{+} e^{-}\right\rangle$, averaged over the initial state polarizations and summed over the final state polarizations, may be written

$$
\begin{aligned}
& \frac{1}{4} \sum_{s_{1}, s_{2}} \sum_{\lambda_{1}, \lambda_{2}}\left|\left\langle W^{+} W^{-}|T| e^{+} e^{-}\right\rangle\right|^{2} \\
& =\frac{1}{4} \sum_{s_{1}, s_{2}}\left(\bar{v} T_{\mu^{\prime} \nu^{\prime}} u\right)^{*}\left(-g^{\mu^{\prime} \mu}+\frac{p_{1}^{\mu^{\prime}} p_{1}^{\mu}}{M_{W}^{2}}\right)\left(-g^{\nu^{\prime} \nu}+\frac{p_{2}^{\nu^{\prime}} p_{2}^{\nu}}{M_{W}^{2}}\right)\left(\bar{v} T_{\mu \nu} u\right) \\
& =\frac{2 \pi^{2} \alpha^{2}}{s_{\mathrm{w}}^{4}} \sum_{i, j} M_{i j}
\end{aligned}
$$


where $i, j=\gamma, Z, \nu$, and $s_{\mathrm{w}}$ is the sine of the weak mixing angle ( $e=g s_{\mathrm{w}}$, where $g$ is the $\mathrm{SU}(2)_{L}$ coupling). In (3.6), the $M_{i j}$ are the various contributions corresponding directly to the diagrams in Fig. 4. For example, $M_{\gamma \gamma}$ is the contribution of the diagram in Fig. 4(a), while $M_{Z \nu}$ is the $Z-\nu$ interference term. These contributions were first calculated almost twenty years ago by Alles, Boyer and Buras, and are given in Eqs. (4.5)-(4.13) of their paper 16. The differential cross section is then given by

$$
\frac{d \sigma\left(e^{+} e^{-} \rightarrow W^{+} W^{-}\right)}{d \Omega}=\frac{\alpha^{2}}{32 s_{\mathrm{w}}^{4}} \beta \frac{1}{s} \sum_{i, j} M_{i j} \equiv \sum_{i, j} \frac{d \sigma_{i j}}{d \Omega} .
$$

Although the individual contributions $d \sigma_{i j} / d \Omega \propto M_{i j}$ in Eq. (3.7) diverge at high energies, the overall differential cross section $d \sigma / d \Omega$ is well behaved as a result of extensive cancellations among the various $d \sigma_{i j} / d \Omega$. However, the bad high energy behaviour of the $d \sigma_{i j} / d \Omega$ precludes the use of the optical theorem (1.1) to interpret individually the components $\left\{\sigma_{\gamma \gamma}, \sigma_{\gamma Z}, \sigma_{Z Z}\right\}$, $\left\{\sigma_{\gamma \nu}, \sigma_{Z \nu}\right\}$ and $\left\{\sigma_{\nu \nu}\right\}$ of the tree level cross section for the process $e^{+} e^{-} \rightarrow W^{+} W^{-}$in terms of the imaginary parts of renormalizable one-loop self-energy-like, vertex-like and box-like $W^{+} W^{-}$contributions, respectively, to the process $e^{+} e^{-} \rightarrow e^{+} e^{-}$.

Here however, rather than decomposing the differential cross section according to the propagator structure of the Green's function $T^{\mu \nu}$, as represented by the diagrams in Fig. 4, we will decompose $d \sigma / d \Omega$ according to the propagator structure occurring in the square of the modulus of the corresponding S-matrix element $i \epsilon_{\mu}^{*} \epsilon_{\nu}^{*} \bar{v} T^{\mu \nu} u$. The crucial observation is that these two decompositions are distinct. This distinction is a fundamental consequence of the non-abelian structure of the electroweak theory, and is encoded in the Ward identity obeyed by $T^{\mu \nu}$. When the factors of longitudinal four-momentum $p_{1 \mu}, p_{2 \nu}$ occurring in the $W^{-}, W^{+}$ polarizations sums in (3.5) are contracted with $T^{\mu \nu}$, this Ward identity i) enforces the exact cancellation among the contributions from diagrams with distinct $s$ - and $t$-channel propagator structure which individually diverge at high energy, and ii) specifies the non-vanishing contributions which remain after this cancellation and which are individually well-behaved at high energy. It is these latter non-vanishing contributions from the longitudinal factors in (3.5), of purely non-abelian origin, Tw which are responsible for the distinction between the pole structure of the Green's function $T^{\mu \nu}$ and that occurring in the square of the modulus of the S-matrix element $i \epsilon_{\mu}^{*} \epsilon_{\nu}^{*} \bar{v} T^{\mu \nu} u$.

The square of the modulus of the S-matrix element in (3.5) may thus be decomposed in terms of a new set of components $\hat{M}_{i j}$, defined as before in terms of the propagator structures which occur, but only after the systematic implementation of the Ward identity obeyed by $T^{\mu \nu}$ and triggered by the factors of longitudinal four-momentum $p_{1 \mu}, p_{2 \nu}$ occurring in the external $W^{-}, W^{+}$polarization vectors. The differential cross section is then given in terms

\footnotetext{
" In QED, the abelian gauge invariance of the theory is such that, for any S-matrix element involving external photons, the Ward identities ensure that the contribution due to a longitudinal factor $p_{\mu}$ associated with the polarization vector $\epsilon_{\mu}(p, \lambda)$ for an external photon vanishes identically.
} 
of the components $\hat{M}_{i j}$ by

$$
\frac{d \sigma\left(e^{+} e^{-} \rightarrow W^{+} W^{-}\right)}{d \Omega}=\frac{\alpha^{2}}{32 s_{\mathrm{w}}^{4}} \beta \frac{1}{s} \sum_{i, j} \hat{M}_{i j} \equiv \sum_{i, j} \frac{d \hat{\sigma}_{i j}}{d \Omega} .
$$

It is emphasized that the decomposition Eq. (3.8) is uniquely specified by the Ward identity for $T^{\mu \nu}$. As will be seen, the decomposition of the tree level cross section for $e^{+} e^{-} \rightarrow W^{+} W^{-}$ in terms of the pole structure occurring in the modulus-squared of the S-matrix element (3.4), rather than that of the corresponding Green's function, will enable the use of the optical theorem (1.1) to interpret individually the various components $\hat{\sigma}_{i j}$ in terms of the imaginary parts of renormalizable one-loop self-energy-like, vertex-like and box-like $W^{+} W^{-}$ contributions, respectively, to the process $e^{+} e^{-} \rightarrow e^{+} e^{-}$.

We begin by decomposing the triple gauge vertex appearing in Figs. 4(a) and (b) as [21]

$$
\Gamma_{\rho \mu \nu}=\Gamma_{\rho \mu \nu}^{F}+\Gamma_{\rho \mu \nu}^{P},
$$

where

$$
\begin{aligned}
& \Gamma_{\rho \mu \nu}^{F}\left(q ;-p_{1},-p_{2}\right)=\left(p_{2}-p_{1}\right)_{\rho} g_{\mu \nu}-2 q_{\mu} g_{\rho \nu}+2 q_{\nu} g_{\rho \mu} \\
& \Gamma_{\rho \mu \nu}^{P}\left(q ;-p_{1},-p_{2}\right)=p_{1 \mu} g_{\rho \nu}-p_{2 \nu} g_{\rho \mu} .
\end{aligned}
$$

The component $\Gamma_{\rho \mu \nu}^{F}$ obeys a simple Ward identity involving the difference of inverse gauge field propagators in the Feynman gauge: $q^{\rho} \Gamma_{\rho \mu \nu}^{F}\left(q ;-p_{1},-p_{2}\right)=\left(p_{2}^{2}-p_{1}^{2}\right) g_{\mu \nu}$. The component $\Gamma_{\rho \mu \nu}^{P}$ vanishes when contracted into the polarization vectors for the on-shell $W^{+} W^{-}$pair: $p_{1}^{\mu} \epsilon_{\mu}\left(p_{1}, \lambda_{1}\right)=p_{2}^{\nu} \epsilon_{\nu}\left(p_{2}, \lambda_{2}\right)=0$.

Choosing to work in the Feynman-'t Hooft gauge, the amputated Green's function represented by the sum of the three diagrams in Fig. 4 may then be decomposed as $[7,8$

$$
T_{\mu \nu}=T_{\mu \nu}^{F}+T_{\mu \nu}^{P}
$$

where

$$
\begin{aligned}
T_{\mu \nu}^{F} & =\left(i e^{2} \gamma^{\rho} \frac{1}{s}+i g^{2} \gamma^{\rho}\left(a-b \gamma_{5}\right) \frac{1}{s-M_{Z}^{2}}\right) \Gamma_{\rho \mu \nu}^{F}-\frac{1}{8} i g^{2} \gamma_{\nu}\left(1-\gamma_{5}\right) \frac{1}{\not k_{1}-\not p_{1}} \gamma_{\mu}\left(1-\gamma_{5}\right), \\
T_{\mu \nu}^{P} & =\left(i e^{2} \gamma^{\rho} \frac{1}{s}+i g^{2} \gamma^{\rho}\left(a-b \gamma_{5}\right) \frac{1}{s-M_{Z}^{2}}\right) \Gamma_{\rho \mu \nu}^{P}
\end{aligned}
$$

$\left(a=\frac{1}{4}-s_{\mathrm{w}}^{2}, b=\frac{1}{4}\right)$. The component $T_{\mu \nu}^{P}$ makes no contribution to the S-matrix element (3.4). Thus, in the expression (3.5), we only need to consider the components $T_{\mu^{\prime} \nu^{\prime}}^{F}, T_{\mu \nu}^{F}$. إ

The next step is to evaluate the effect of the factors of longitudinal four-momenta in Eq. (3.5). For a factor $p_{1}^{\mu}$, using the tree level Ward identities

$$
\begin{aligned}
p_{1}^{\mu} \Gamma_{\rho \mu \nu}^{F}\left(q ;-p_{1},-p_{2}\right) & =-\left(s+p_{1}^{2}-p_{2}^{2}\right) g_{\rho \nu}+q_{\rho} q_{\nu}+\left(p_{1}-p_{2}\right)_{\rho} p_{2 \nu}, \\
p_{1}^{\mu} \gamma_{\mu} & =k_{1}-\left(k_{1}-\not p_{1}\right),
\end{aligned}
$$

\footnotetext{
"It is worth remarking that the decomposition (3.12) is not necessary, but simply facilitates the calculation of the cross section.
} 


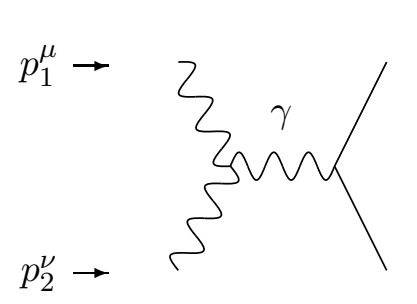

(a)

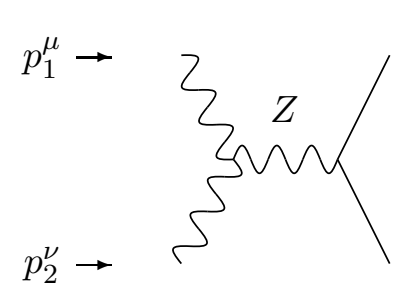

(b)

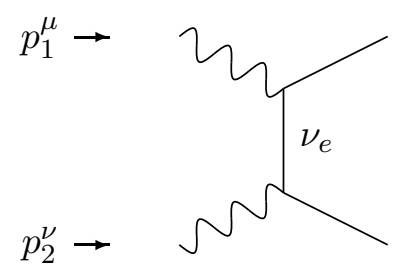

(c)

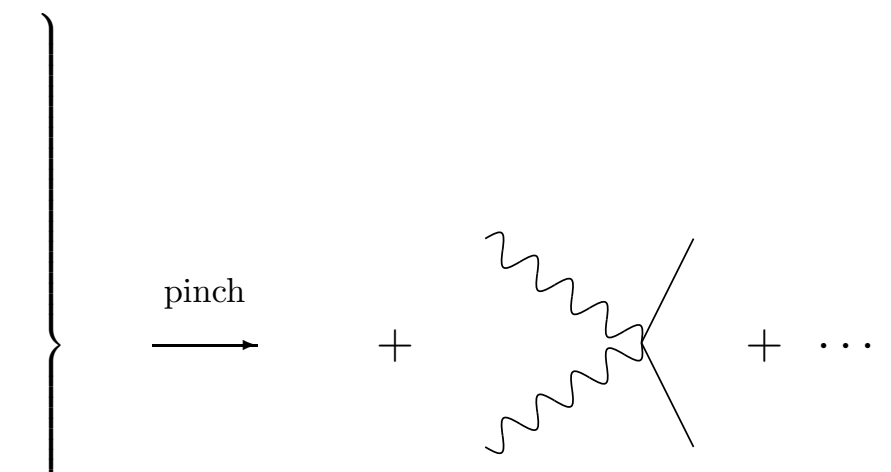

(d)

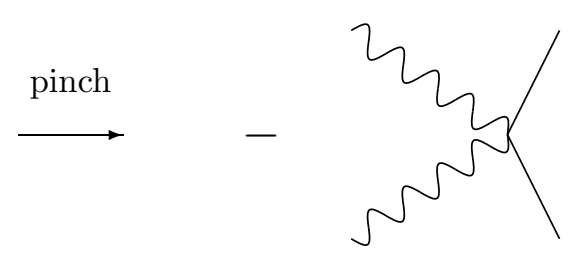

(e)

Fig. 5. The diagrammatic representation of the cancellation between i) the component of the diagrams (a) and (b) in which the $\gamma$ and $Z$ propagators have been cancelled (pinched) by the action of the factors of longitudinal four-momentum $p_{1}^{\mu}, p_{2}^{\nu}$ appearing in the $W^{-}, W^{+}$polarization sums in Eq. (3.5), and ii) the entire contribution of the diagram (c), in which the $\nu_{e}$ propagator has been cancelled (pinched) by the same factors. The ellipsis in diagram (d) represents the remaining terms.

together with the fact that the external fields are on-shell (so that $\bar{v}\left(k_{2}, s_{2}\right) k_{2}=k_{1} u\left(k_{1}, s_{1}\right)=$ 0 and $p_{1}^{2}=p_{2}^{2}=M_{W}^{2}$ ), we obtain

$$
\begin{aligned}
p_{1}^{\mu} \bar{v} T_{\mu \nu}^{F} u & =g s_{\mathrm{w}}\left(-i e \bar{v} \gamma^{\rho} u\right) \frac{1}{s}\left(p_{2}-p_{1}\right)_{\rho} p_{2 \nu} \\
& +g c_{\mathrm{w}}\left(-i\left(g / c_{\mathrm{w}}\right) \bar{v} \gamma^{\rho}\left(a-b \gamma_{5}\right) u\right) \frac{1}{s-M_{Z}^{2}}\left(M_{Z}^{2} g_{\nu \rho}+\left(p_{2}-p_{1}\right)_{\rho} p_{2 \nu}\right) .
\end{aligned}
$$

The simple dependence of this expression on the $s$-channel $\gamma$ and $Z$ propagators and the corresponding ee $\gamma$ and $e e Z$ couplings is apparent. The crucial feature of the Ward identity Eq. (3.17) is the exact cancellation which has occurred between i) components of the $\gamma$ and $Z$ contributions to $T_{\mu \nu}^{F}$ in Eq. (3.13) in which the $\gamma$ and $Z$ propagators have been cancelled (pinched) by the term $s g_{\rho \nu}$ in Eq. (3.15), and ii) the entire $\nu$ contribution to $T_{\mu \nu}^{F}$ in which the $\nu_{e}$ propagator has been cancelled (pinched) by the term $k_{1}-\not \phi_{1}$ in Eq. (3.16). This cancellation is illustrated in Fig. 5. 
Using the Ward identity Eq. (3.17) together with the similar expressions for the various other longitudinal factors which occur in Eq. (3.5), we can isolate the contributions to the cross section according to the $\gamma, Z$ and $\nu$ propagator structure occurring in the square of the modulus of the S-matrix element, as described above. In the centre of mass frame we obtain:

$$
\begin{aligned}
& \hat{M}_{\gamma \gamma}=s_{\mathrm{w}}^{4}\left\{12 \beta^{2} \sin ^{2} \theta-64\right\} \\
& \hat{M}_{\gamma Z}=2 s_{\mathrm{w}}^{2} a \frac{s}{\left(s-M_{Z}^{2}\right)}\left\{\left(12-2 \frac{1}{c_{\mathrm{w}}^{2}}\right) \beta^{2} \sin ^{2} \theta-64\right\} \\
& \hat{M}_{Z Z}=\left(a^{2}+b^{2}\right) \frac{s^{2}}{\left(s-M_{Z}^{2}\right)^{2}}\left\{\left(12-4 \frac{1}{c_{\mathrm{w}}^{2}}+\frac{1}{c_{\mathrm{w}}^{4}}\right) \beta^{2} \sin ^{2} \theta-64+16 \frac{1}{c_{\mathrm{w}}^{4}} \frac{M_{W}^{2}}{s}\right\} ; \\
& \hat{M}_{\gamma \nu}=2 s_{\mathrm{w}}^{2} \frac{s}{t}\left\{\beta^{2} \sin ^{2} \theta-4(1-\beta \cos \theta)\right\} \\
& \hat{M}_{Z \nu}=2(a+b) \frac{s}{\left(s-M_{Z}^{2}\right)} \frac{s}{t}\left\{\beta^{2} \sin ^{2} \theta-4(1-\beta \cos \theta)\right\} ; \\
& \hat{M}_{\nu \nu}=\frac{1}{2} \frac{s^{2}}{t^{2}} \beta^{2} \sin ^{2} \theta .
\end{aligned}
$$

The above expressions have been grouped into self-energy-like, vertex-like and box-like contributions according to the presence of zero, one or two internal $\nu_{e}$ propagators, respectively. In each case, an overall factor $\vartheta\left(s-4 M_{W}^{2}\right)$ has been omitted for brevity.

Carrying out the angular integrations, the corresponding contributions to the total cross section are given by

$$
\begin{aligned}
& \hat{\sigma}_{\gamma \gamma}=2 \pi \alpha^{2} \frac{1}{s} \beta\left\{-\frac{7}{2}-\frac{2}{y}\right\} \\
& \hat{\sigma}_{\gamma Z}=4 \pi \alpha^{2} \frac{a}{s_{\mathrm{w}}^{2} c_{\mathrm{w}}^{2}} \frac{1}{\left(s-M_{Z}^{2}\right)} \beta\left\{-\frac{43}{12}-\frac{5}{3 y}-s_{\mathrm{w}}^{2}\left(-\frac{7}{2}-\frac{2}{y}\right)\right\} \\
& \hat{\sigma}_{Z Z}=2 \pi \alpha^{2} \frac{\left(a^{2}+b^{2}\right)}{s_{\mathrm{w}}^{4} c_{\mathrm{w}}^{4}} \frac{s}{\left(s-M_{Z}^{2}\right)^{2}} \beta\left\{-\frac{29}{8}-\frac{1}{2 y}-2 s_{\mathrm{w}}^{2}\left(-\frac{43}{12}-\frac{5}{3 y}\right)+s_{\mathrm{w}}^{4}\left(-\frac{7}{2}-\frac{2}{y}\right)\right\} \\
& \hat{\sigma}_{\gamma \nu}=4 \pi \alpha^{2} \frac{1}{s_{\mathrm{w}}^{2}} \frac{1}{s} \beta\left\{\left(\frac{1}{y}+\frac{1}{2 y^{2}}\right) \frac{L}{\beta}+\frac{3}{8}+\frac{1}{4 y}\right\} \\
& \hat{\sigma}_{Z \nu}=4 \pi \alpha^{2} \frac{(a+b)}{s_{\mathrm{w}}^{4}} \frac{1}{\left(s-M_{Z}^{2}\right)} \beta\left\{\left(\frac{1}{y}+\frac{1}{2 y^{2}}\right) \frac{L}{\beta}+\frac{3}{8}+\frac{1}{4 y}\right\} \\
& \hat{\sigma}_{\nu \nu}=2 \pi \alpha^{2} \frac{1}{s_{\mathrm{w}}^{4}} \frac{1}{s} \beta\left\{\left(\frac{1}{4}-\frac{1}{2 y}\right) \frac{L}{\beta}-\frac{1}{4}\right\} .
\end{aligned}
$$

Here $y \equiv s / M_{W}^{2}, L \equiv \ln [(1+\beta) /(1-\beta)]$ and again the factor $\vartheta\left(s-4 M_{W}^{2}\right)$ is omitted. 


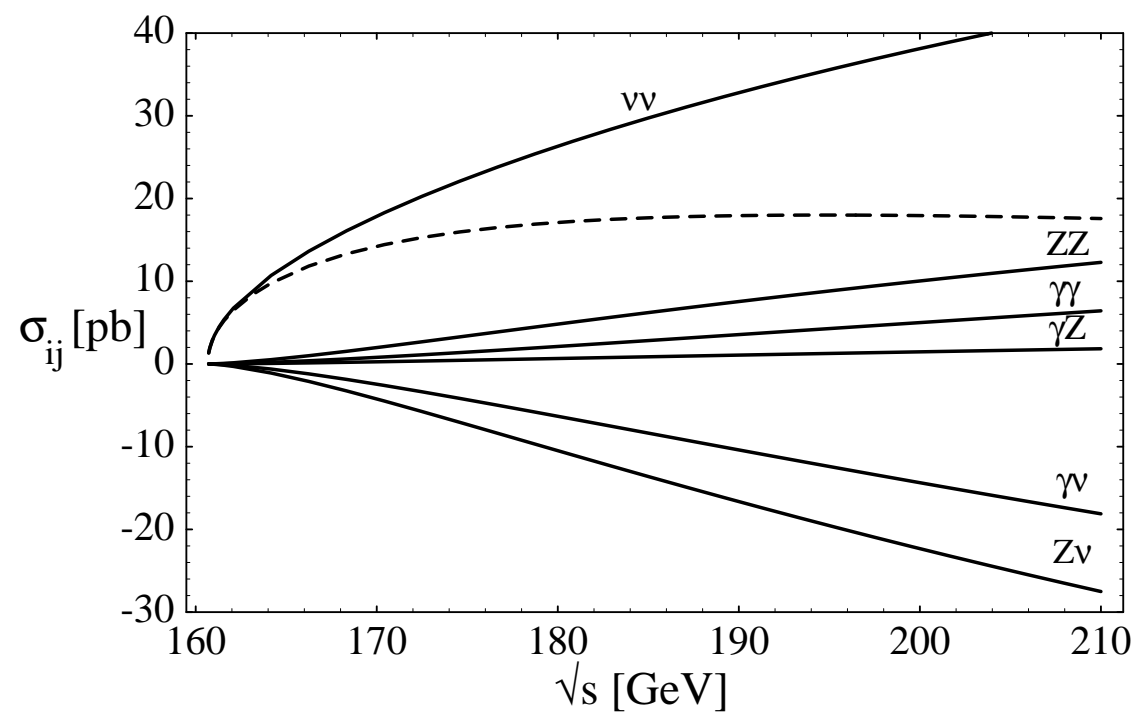

Fig. 6(a). The components $\sigma_{i j}, i, j=\gamma, Z, \nu$, of the tree level cross section $\sigma\left(e^{+} e^{-} \rightarrow W^{+} W^{-}\right)$, corresponding to the decomposition directly in terms of the diagrams in Fig. 4, taken from Ref. [16]. The dashed line is the full cross section, given by the sum of the components.

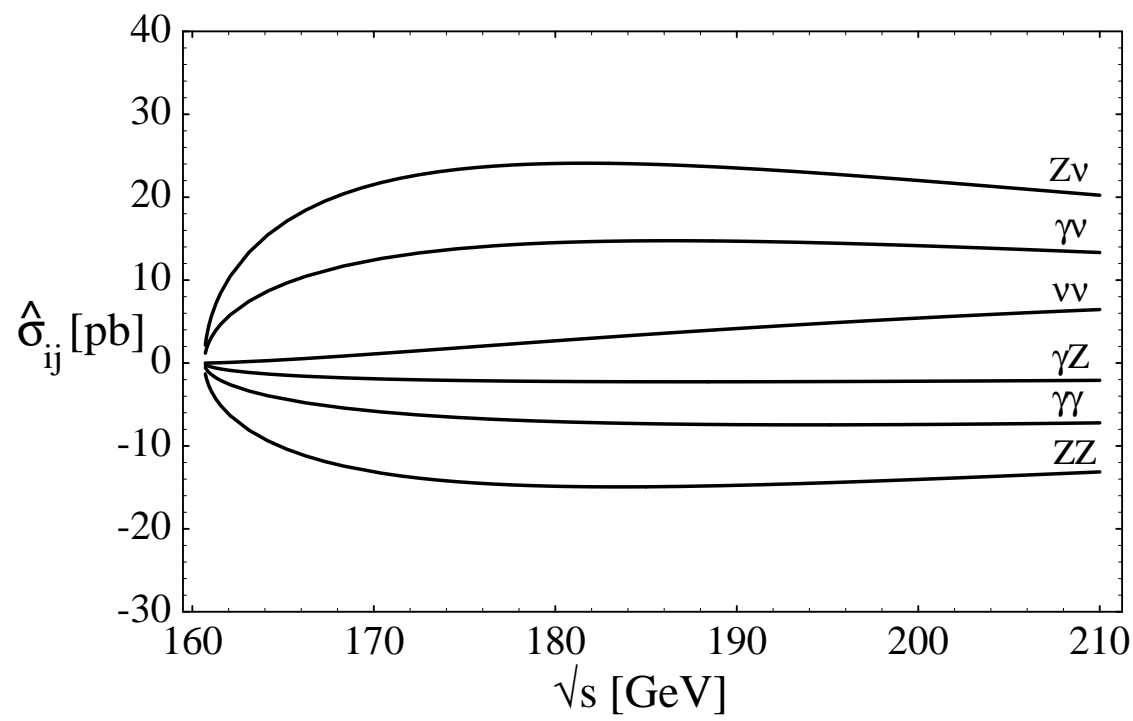

Fig. 6(b). The components $\hat{\sigma}_{i j}, i, j=\gamma, Z, \nu$, of the tree level cross section $\sigma\left(e^{+} e^{-} \rightarrow W^{+} W^{-}\right)$, corresponding to the decomposition according to the propagator structure occurring in the square of the modulus of the S-matrix element, and given in Eqs. (3.24)-(3.29).

The cross section components $\hat{\sigma}_{i j}$ given in Eqs. (3.24)-(3.29) are shown plotted in Fig. 6(b). These plots are to be compared with the plots of the components $\sigma_{i j}$ obtained by Alles, Boyer and Buras [16] shown in Fig. 6(a). 
Several remarks are in order:

- It is easily verified that these expressions Eqs. (3.18)-(3.23) and (3.24)-(3.29) combine to reproduce, respectively, the correct differential and total cross sections given in [16.

- At asymptotic $s$, each of the contributions $\hat{M}_{i j} \sim$ const. This is in contrast to the conventional decomposition, in which, as a result of the longitudinal factors in the $W^{+}$, $W^{-}$polarization sums, each of the $M_{i j} \sim s^{2}$ so that individually they violate unitarity; it is only when summed that, as a result of cancellations, $\sum_{i j} M_{i j} \sim$ const. and unitarity is restored [22, 23]. Here, by systematically implementing on the r.h.s. of Eq. (3.5) the Ward identity Eq. (3.17) triggered by these longitudinal factors, this cancellation has occurred at the level of the individual components $\hat{M}_{i j}$ of the differential cross section rather than the overall differential cross section itself.

- The three self-energy-like cross section contributions $\hat{\sigma}_{\gamma \gamma}, \hat{\sigma}_{\gamma Z}$ and $\hat{\sigma}_{Z Z}$ are each negative. This is directly related to the fact that the $W^{ \pm}$gauge boson contributions to the one-loop electroweak $\beta$-functions are negative. In particular, the coefficient $-\frac{7}{2}=-\frac{11}{6} \times 2+\frac{1}{6}$ in the expression Eq. (3.24) for $\hat{\sigma}_{\gamma \gamma}$ is precisely the one-loop contribution of the massive $W^{ \pm}$gauge bosons to the $\beta$-function for the electromagnetic coupling $\alpha$. Similarly, writing $J_{\gamma} J_{Z}=\left(J_{3}+\frac{1}{2} J_{Y}\right) J_{3}-s_{\mathrm{w}}^{2} J_{\gamma} J_{\gamma}$ where $J_{\gamma}, J_{Z}, J_{3}$ and $J_{Y}$ are the currents which couple to the $\gamma, Z, W_{3}$ and $B$ gauge fields respectively ( $W_{3}$ is the neutral component of the $\mathrm{SU}(2)_{L}$ triplet, while $B$ is the $\mathrm{U}(1)_{Y}$ singlet), the coefficient $-\frac{43}{12}=-\frac{11}{6} \times 2+\frac{1}{12}$ in the expression Eq. (3.25) for $\hat{\sigma}_{\gamma Z}$ is the one-loop contribution of the massive $W^{ \pm}$gauge bosons to the $\beta$-function for the $\mathrm{SU}(2)_{L}$ coupling $g^{2} / 4 \pi$. In each case, the term $-\frac{11}{6} \times 2$ is the pure gauge field contribution, while the terms $+\frac{1}{6}$ and $+\frac{1}{12}$ respectively are the contributions of the complex doublet of scalars involved in the spontaneous symmetry breaking (cf. Sec. 6). The appearance of the $W^{ \pm}$gauge boson contributions to the electroweak $\beta$-functions in the $e^{+} e^{-} \rightarrow W^{+} W^{-}$cross section components $\hat{\sigma}_{\gamma \gamma}$ and $\hat{\sigma}_{\gamma Z}$ is exactly analogous to the appearance of the $f^{ \pm}$fermion contribution to the QED $\beta$-function in the $e^{+} e^{-} \rightarrow f^{+} f^{-}$cross section Eq. (2.3).

\section{$3.2 e^{+} e^{-} \rightarrow Z H$}

We next consider the process $e^{-}\left(k_{1}, s_{1}\right) e^{+}\left(k_{2}, s_{2}\right) \rightarrow Z\left(p_{1}, \lambda_{1}\right) H\left(p_{2}\right)$ at tree level, where $H$ is the Higgs scalar. Neglecting the fermion mass, the only diagram which contributes to this process is that shown in Fig. 7.

A straightforward calculation gives for the cross section

$$
\sigma\left(e^{+} e^{-} \rightarrow Z H\right)=2 \pi \alpha^{2} \frac{\left(a^{2}+b^{2}\right)}{s_{\mathrm{w}}^{4} c_{\mathrm{w}}^{4}} \frac{s}{\left(s-M_{Z}^{2}\right)^{2}} \lambda^{\frac{1}{2}}\left(s, M_{Z}, M_{H}\right)\left\{\frac{1}{24}+\frac{5 M_{Z}^{2}}{12 s}-\frac{M_{H}^{2}}{12 s}+\frac{\left(M_{Z}^{2}-M_{H}^{2}\right)^{2}}{24 s^{2}}\right\}
$$

where $M_{H}$ is the Higgs mass and $\lambda\left(s, M_{i}, M_{j}\right)=\left(1-\left(M_{i}+M_{j}\right)^{2} / s\right)\left(1-\left(M_{i}-M_{j}\right)^{2} / s\right)$. 


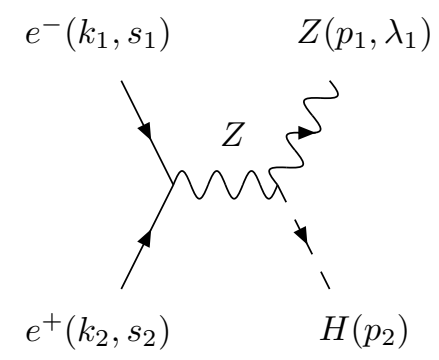

Fig. 7. The single diagram which contributes to the process $e^{+} e^{-} \rightarrow Z H$ at tree level for the case of massless fermions.

Two remarks are in order:

- The calculation of the cross section Eq. 3.30 involves only a single diagram, so that, in contrast to the process $e^{+} e^{-} \rightarrow W^{+} W^{-}$, there is no cancellation to account for involving factors of external gauge field longitudinal four-momentum.

- In the high energy limit $s / M_{Z}^{2} \rightarrow \infty$, the sum of the $e^{+} e^{-} \rightarrow W^{+} W^{-}$self-energy-like cross section component $\hat{\sigma}_{Z Z}$ Eq. (3.26) and the $e^{+} e^{-} \rightarrow Z H$ cross section Eq. (3.30) is given by

$$
\begin{aligned}
& \hat{\sigma}_{Z Z}\left(e^{+} e^{-} \rightarrow W^{+} W^{-}\right)+\sigma\left(e^{+} e^{-} \rightarrow Z H\right)= \\
& 2 \pi \alpha^{2} \frac{\left(a^{2}+b^{2}\right)}{s_{\mathrm{w}}^{4} c_{\mathrm{w}}^{4}} \frac{1}{s}\left\{-\frac{43}{12}-2 s_{\mathrm{w}}^{2}\left(-\frac{43}{12}\right)+s_{\mathrm{w}}^{4}\left(-\frac{7}{2}\right)+\mathcal{O}\left(\frac{M_{Z}^{2}}{s}, \frac{M_{H}^{2}}{s}\right)\right\}
\end{aligned}
$$

Writing $J_{Z} J_{Z}=J_{3} J_{3}-2 s_{\mathrm{w}}^{2}\left(J_{3}+\frac{1}{2} J_{Y}\right) J_{3}+s_{\mathrm{w}}^{4} J_{\gamma} J_{\gamma}$, the coefficient $-\frac{43}{12}=-\frac{29}{8}+\frac{1}{24}=$ $-\frac{11}{6} \times 2+\frac{1}{12}$ in Eq. (3.31) is again precisely the one-loop contribution of the massive electroweak gauge bosons to the $\beta$-function for the $\mathrm{SU}(2)_{L}$ coupling $g^{2} / 4 \pi$ (cf. Sec. 6 ).

\section{The Electroweak Gauge Boson Self-Energies}

We now turn to the gauge boson self-energies. In general, the transverse self-energy function $\Sigma_{i j}$ for gauge bosons $i, j$ requires two subtractions, for mass and field renormalization**: with subtraction point $s_{i j}$, the renormalized self-energy $\Sigma_{R, i j}$ is given by

$$
\begin{aligned}
\Sigma_{R, i j}\left(q^{2}\right) & =\Sigma_{i j}\left(q^{2}\right)-\Sigma_{i j}\left(s_{i j}\right)-\left.\left(q^{2}-s_{i j}\right) \frac{\partial \Sigma_{i j}\left(q^{2}\right)}{\partial q^{2}}\right|_{s_{i j}} \\
& =\left(q^{2}-s_{i j}\right)^{2} \int_{0}^{\infty} d s \frac{1}{\left(s-q^{2}\right)\left(s-s_{i j}\right)^{2}} \frac{1}{\pi} \operatorname{Im} \Sigma_{i j}(s)
\end{aligned}
$$

** For a thorough review of radiative corrections in electroweak theory, where many references may be found, see e.g. Ref. 24. 
In the case where $s_{i j}=0$ and $\Sigma_{i j}(0)=0$, writing $\Sigma_{i j}\left(q^{2}\right)=q^{2} \Pi_{i j}\left(q^{2}\right)$, the twice-subtracted dispersion relation Eq. (4.2) reduces to a once-subtracted expression identical to Eq. (2.4) for the QED vacuum polarization renormalized in the on-shell scheme.

In the conventional perturbation theory approach, the gauge boson plus associated wouldbe Goldstone boson and ghost contributions to the electroweak self-energies $\Sigma_{i j}$ are gaugedependent (for expressions in the class of renormalizable gauges, see e.g. Refs. 225]). Here however, rather than considering the contributions to $\Sigma_{i j}$ from one-loop perturbation theory diagrams in a given gauge, we will follow the dispersive procedure described in Sec. 2 for the case of QED to define one-loop electroweak self-energy-like functions $\hat{\Sigma}_{i j}$ directly from the tree level cross sections for physical processes. In particular, having decomposed the tree level cross section for $e^{+} e^{-} \rightarrow W^{+} W^{-}$in terms of the pole structure occurring in the square of the modulus of the corresponding $\mathrm{S}$-matrix element, we define the imaginary parts of the $W^{+} W^{-}$contributions to the $\hat{\Sigma}_{i j}$ via

$$
\begin{aligned}
e^{2} \frac{1}{s^{2}} \operatorname{Im} \hat{\Sigma}_{\gamma \gamma}^{(W W)}(s) & =\hat{\sigma}_{\gamma \gamma}\left(e^{+} e^{-} \rightarrow W^{+} W^{-}\right), \\
2 e^{2} \frac{a}{s_{\mathrm{w}} c_{\mathrm{w}}} \frac{1}{s\left(s-M_{Z}^{2}\right)} \operatorname{Im} \hat{\Sigma}_{\gamma Z}^{(W W)}(s) & =\hat{\sigma}_{\gamma Z}\left(e^{+} e^{-} \rightarrow W^{+} W^{-}\right), \\
e^{2} \frac{\left(a^{2}+b^{2}\right)}{s_{\mathrm{w}}^{2} c_{\mathrm{w}}^{2}} \frac{1}{\left(s-M_{Z}^{2}\right)^{2}} \operatorname{Im} \hat{\Sigma}_{Z Z}^{(W W)}(s) & =\hat{\sigma}_{Z Z}\left(e^{+} e^{-} \rightarrow W^{+} W^{-}\right) .
\end{aligned}
$$

Similarly, we define the imaginary part of the $Z H$ contribution to $\hat{\Sigma}_{Z Z}$ via

$$
e^{2} \frac{\left(a^{2}+b^{2}\right)}{s_{\mathrm{w}}^{2} c_{\mathrm{w}}^{2}} \frac{1}{\left(s-M_{Z}^{2}\right)^{2}} \operatorname{Im} \hat{\Sigma}_{Z Z}^{(Z H)}(s)=\sigma\left(e^{+} e^{-} \rightarrow Z H\right) .
$$

These definitions are by direct analogy with the QED relation Eq. (2.2) (with $m_{e}=0$ ) between the imaginary part of the fermion contribution to the one-loop photon self-energy $\Sigma\left(q^{2}\right)=q^{2} \Pi\left(q^{2}\right)$ and the tree level cross section for $e^{+} e^{-} \rightarrow f^{+} f^{-}$: in each case there is a factor consisting of the product of propagators $\left(q^{2}-M_{i}^{2}\right)^{-1}\left(q^{2}-M_{j}^{2}\right)^{-1}$ multiplying the couplings of the corresponding gauge fields $i, j$ to the $e^{+} e^{-}$pair. Given the contribution of a particular pair of fields to $\operatorname{Im} \hat{\Sigma}_{i j}$ together with an appropriate choice for the subtraction point $s_{i j}$, the contribution of the fields to the renormalized self-energy function $\hat{\Sigma}_{R, i j}$ can then be reconstructed using the dispersion relation Eq. (4.2).

It is emphasized that the analogy with the QED relation Eq. (2.2) uniquely specifies the $W^{+} W^{-}$contributions to the one-loop self-energies in terms of the self-energy-like components of the tree level $e^{+} e^{-} \rightarrow W^{+} W^{-}$cross section, which are in turn uniquely specified by the systematic and exhaustive implementation of the Ward identity for the corresponding tree level amputated Green's function, as described in Sec. 3.1. In particular, the renormalized self-energies $\hat{\Sigma}_{i j}$ defined in this way in terms of the cross sections for physical processes are clearly gauge-independent. Furthermore, we will show how, by defining the electroweak selfenergies by direct analogy with the properties (ii) and (iii) of the QED self-energy described 
in Sec. 2, not only does the basic QED property (i) of gauge independence extend to the electroweak theory (to one-loop level), but also each of the further properties (iv)-(vii).

We now choose the on-shell renormalization scheme. The subtraction points are then $s_{\gamma \gamma}=s_{\gamma Z}=0$ and $s_{Z Z}=M_{Z}^{2}$. Using the definitions Eqs. (4.3)-(4.5), the imaginary parts of the $W^{+} W^{-}$contributions to $\hat{\Sigma}_{\gamma \gamma}, \hat{\Sigma}_{\gamma Z}$ and $\hat{\Sigma}_{Z Z}$ may be read off from the $e^{+} e^{-} \rightarrow W^{+} W^{-}$ cross section contributions $\hat{\sigma}_{\gamma \gamma}, \hat{\sigma}_{\gamma Z}$ and $\hat{\sigma}_{Z Z}$, respectively, given in Eqs. (3.24)-(3.26). Inserting these expressions in the dispersion relation Eq. (4.2) and carrying out the integrations, we obtain:

$$
\begin{aligned}
\hat{\Sigma}_{R, \gamma \gamma}^{(W W)}\left(q^{2}\right) & =\frac{\alpha}{\pi} q^{2}\left\{\left(\frac{7}{2}+\frac{2 M_{W}^{2}}{q^{2}}\right) B\left(q^{2}\right)+\frac{1}{6}\right\} \\
\hat{\Sigma}_{R, \gamma Z}^{(W W)}\left(q^{2}\right) & =\frac{\alpha}{\pi} \frac{1}{s_{\mathrm{w}} c_{\mathrm{w}}} q^{2}\left\{\left[\left(\frac{43}{12}+\frac{5 M_{W}^{2}}{3 q^{2}}\right)-s_{\mathrm{w}}^{2}\left(\frac{7}{2}+\frac{2 M_{W}^{2}}{q^{2}}\right)\right] B\left(q^{2}\right)+\frac{5}{36}-s_{\mathrm{w}}^{2} \frac{1}{6}\right\},(4.8 \\
\hat{\Sigma}_{R, Z Z}^{(W W)}\left(q^{2}\right) & =\frac{\alpha}{\pi} \frac{1}{s_{\mathrm{w}}^{2} c_{\mathrm{w}}^{2}}\left(q^{2}-M_{Z}^{2}\right) \times \\
\{ & {\left[\left(\frac{29}{8}+\frac{M_{W}^{2}}{2 q^{2}}\right)-2 s_{\mathrm{w}}^{2}\left(\frac{43}{12}+\frac{5 M_{W}^{2}}{3 q^{2}}\right)+s_{\mathrm{w}}^{4}\left(\frac{7}{2}+\frac{2 M_{W}^{2}}{q^{2}}\right)\right] q^{2}\left(\frac{B\left(q^{2}\right)-B\left(M_{Z}^{2}\right)}{q^{2}-M_{Z}^{2}}\right) } \\
- & {\left.\left.\left[\left(\frac{29}{8}+\frac{1}{2} c_{\mathrm{w}}^{2}\right)-2 s_{\mathrm{w}}^{2}\left(\frac{43}{12}+\frac{5}{3} c_{\mathrm{w}}^{2}\right)+s_{\mathrm{w}}^{4}\left(\frac{7}{2}+2 c_{\mathrm{w}}^{2}\right)\right] M_{Z}^{2} \frac{d B\left(q^{2}\right)}{d q^{2}}\right|_{M_{Z}^{2}}\right\}, }
\end{aligned}
$$

where the function $B\left(q^{2}\right)$ is given by

$$
B\left(q^{2}\right)=\frac{\beta}{2} \ln \left(\frac{\beta+1}{\beta-1}\right)-1, \quad \beta=\sqrt{1-\frac{4 M_{W}^{2}}{q^{2}}} .
$$

Thus,

$$
\begin{aligned}
\operatorname{Im} B\left(q^{2}\right) & =-\frac{\pi}{2} \beta \vartheta\left(q^{2}-4 M_{W}^{2}\right) \\
q^{2} \frac{d B\left(q^{2}\right)}{d q^{2}} & =\frac{1}{\beta^{2}}\left(\frac{2 M_{W}^{2}}{q^{2}} B\left(q^{2}\right)+\frac{1}{2}\right) .
\end{aligned}
$$

For asymptotic $q^{2}$,

$$
B\left(q^{2}\right)=\left\{\begin{array}{lr}
-\frac{1}{12} \frac{q^{2}}{M_{W}^{2}}-\frac{1}{120} \frac{q^{4}}{M_{W}^{4}}+\mathcal{O}\left(\frac{q^{6}}{M_{W}^{6}}\right) & q^{2} / M_{W}^{2} \rightarrow 0 \\
\frac{1}{2} \ln \left(-\frac{q^{2}}{M_{W}^{2}}\right)-1+\mathcal{O}\left(\frac{M_{W}^{2}}{q^{2}}\right) & -q^{2} / M_{W}^{2} \rightarrow \infty .
\end{array}\right.
$$

Similarly, using the definition Eq. (4.6), the imaginary part of the $Z H$ contribution to $\hat{\Sigma}_{R, Z Z}$ may be read off from the cross section for $e^{+} e^{-} \rightarrow Z H$ given in Eq. (3.30). The function $\hat{\Sigma}_{R, Z Z}^{(Z H)}\left(q^{2}\right)$ may then be obtained from the dispersion relation Eq. (4.2). The resulting expression is given in App. A.

The expressions Eqs. (4.7)-4.9) for $\hat{\Sigma}_{R, \gamma \gamma}^{(W W)}, \hat{\Sigma}_{R, \gamma Z}^{(W W)}$ and $\hat{\Sigma}_{R, Z Z}^{(W W)}$ and Eq. (A.8) for $\hat{\Sigma}_{R, Z Z}^{(Z H)}$ are identical to the corresponding renormalized gauge-independent self-energy contributions 
obtained in the pinch technique 13]. In the usual pinch technique approach, these self-energy contributions were obtained via the rearrangement of conventional one-loop perturbation theory diagrams. Here, we have obtained exactly the same results directly from components of the tree level cross sections for physical processes.

An exactly similar procedure to that described above can be carried out for the charged current interactions in order to define the gauge boson contributions to the renormalized one-loop $W$ self-energy $\hat{\Sigma}_{R, W W}$. The relation between the imaginary part of $\hat{\Sigma}_{R, W W}$ and the tree level cross sections for the corresponding physical processes is specified by the optical theorem for the case of forward scattering in the process $e^{+} \nu_{e} \rightarrow e^{+} \nu_{e}$ :

$$
\operatorname{Im}\left\langle e^{+} \nu_{e}|T| e^{+} \nu_{e}\right\rangle=\frac{1}{2} \sum_{i} \int d \Gamma_{i}\left|\left\langle e^{+} \nu_{e}|T| i\right\rangle\right|^{2} .
$$

The on-shell states involving the electroweak gauge bosons $W$ and $Z$ which contribute to the r.h.s. of Eq. (4.14) at lowest order are $\left|W^{+} Z\right\rangle,\left|W^{+} \gamma\right\rangle$ and $\left|W^{+} H\right\rangle$, corresponding to the tree level physical processes $e^{+} \nu_{e} \rightarrow W^{+} Z, e^{+} \nu_{e} \rightarrow W^{+} \gamma$ and $e^{+} \nu_{e} \rightarrow W^{+} H$.

For the processes $e^{+} \nu_{e} \rightarrow W^{+} Z$ and $e^{+} \nu_{e} \rightarrow W^{+} \gamma$, an exactly similar cancellation occurs among contributions to the cross section involving factors of external gauge field longitudinal four-momentum as occurred in the process $e^{+} e^{-} \rightarrow W^{+} W^{-}$analysed in Sec. 3.1. In each case, the square of the modulus of the S-matrix element may be decomposed according to the pole structure which occurs after the systematic implementation of the Ward identity obeyed by the corresponding Green's function. In particular, the self-energy-like components $\hat{\sigma}_{W W}\left(e^{+} \nu_{e} \rightarrow W^{+} Z\right)$ and $\hat{\sigma}_{W W}\left(e^{+} \nu_{e} \rightarrow W^{+} \gamma\right)$ of the two cross sections are the contributions after cancellations which involve a pair of $s$-channel $W$ propagators $\left(s-M_{W}^{2}\right)^{-1}$. The imaginary parts of the $W Z$ and $W \gamma$ contributions to the $W$ self-energy $\hat{\Sigma}_{W W}$ are then defined via $\left(m_{e}=0\right)$

$$
\begin{aligned}
& e^{2} \frac{1}{4 s_{\mathrm{w}}^{2}} \frac{1}{\left(s-M_{W}^{2}\right)^{2}} \operatorname{Im} \hat{\Sigma}_{W W}^{(W Z)}(s)=\hat{\sigma}_{W W}\left(e^{+} \nu_{e} \rightarrow W^{+} Z\right), \\
& e^{2} \frac{1}{4 s_{\mathrm{w}}^{2}} \frac{1}{\left(s-M_{W}^{2}\right)^{2}} \operatorname{Im} \hat{\Sigma}_{W W}^{(W \gamma)}(s)=\hat{\sigma}_{W W}\left(e^{+} \nu_{e} \rightarrow W^{+} \gamma\right) .
\end{aligned}
$$

For the process $e^{+} \nu_{e} \rightarrow W^{+} H$, involving only a single diagram at tree level, there is no cancellation to account for. The imaginary part of the $W H$ contribution to $\hat{\Sigma}_{W W}$ is defined via

$$
e^{2} \frac{1}{4 s_{\mathrm{w}}^{2}} \frac{1}{\left(s-M_{W}^{2}\right)^{2}} \operatorname{Im} \hat{\Sigma}_{W W}^{(W H)}(s)=\sigma\left(e^{+} \nu_{e} \rightarrow W^{+} H\right) .
$$

In the on-shell renormalization scheme, the subtraction point $s_{W W}=M_{W}^{2}$. The $W Z$, $W \gamma$ and $W H$ contributions to the renormalized one-loop $W$ self-energy $\hat{\Sigma}_{W W}$ can then be reconstructed from the expressions for $\hat{\sigma}_{W W}\left(e^{+} \nu_{e} \rightarrow W^{+} Z\right), \hat{\sigma}_{W W}\left(e^{+} \nu_{e} \rightarrow W^{+} \gamma\right)$ and $\sigma\left(e^{+} \nu_{e} \rightarrow W^{+} H\right)$, respectively, using the dispersion relation Eq. (4.2) in conjunction with the above definitions. The resulting expressions for the functions $\hat{\Sigma}_{W W}^{(W Z)}, \hat{\Sigma}_{W W}^{(W \gamma)}$ and $\hat{\Sigma}_{W W}^{(W H)}$ 
are given in App. A. These functions are again identical to the corresponding renormalized gauge-independent self-energy contributions obtained in the pinch technique [13].

It is important to point out that the PT one-loop gauge boson self-energy functions are universal [14]. This is a direct result of the tree level Ward identities of the given theory [7, 8]. In the approach followed here, this universality corresponds to the fact that the imaginary parts of the one-loop contributions of fields $C, D$ to the PT self-energies may be obtained from the self-energy-like components of the tree level cross section $\sigma(A B \rightarrow C D)$ for the interaction of any pair of fields $A, B$ to which the gauge bosons couple at tree level. For example, the $W^{+} W^{-}$contributions to $\hat{\Sigma}_{\gamma \gamma}, \hat{\Sigma}_{\gamma Z}$ and $\hat{\Sigma}_{Z Z}$ may just as well be obtained from the self-energy-like components of the cross section $\sigma\left(W^{+} W^{-} \rightarrow W^{+} W^{-}\right)$as from the cross section $\sigma\left(e^{+} e^{-} \rightarrow W^{+} W^{-}\right)$considered here.

\section{The Electroweak Effective Charges}

We next turn to the electroweak effective charges. Beyond tree level, the $\gamma-Z$ mixing induced by the $\hat{\Sigma}_{\gamma Z}$ self-energy requires the neutral current sector to be re-diagonalized. In Refs. [6, 8], it has been shown how the pinch technique gauge boson self-energies may be summed in Dyson series, in exactly the same way as the conventional gauge-dependent self-energies. We can therefore follow the standard diagonalization procedure [26] except for the use of PT self-energies in place of the conventional functions.

The PT bare neutral current two-point functions can be written in matrix form as

$$
\hat{\Gamma}_{N C}=\left(\begin{array}{cc}
q^{2}+\hat{\Sigma}_{\gamma \gamma} & \hat{\Sigma}_{\gamma Z} \\
\hat{\Sigma}_{\gamma Z} & q^{2}-M_{Z}^{2}+\hat{\Sigma}_{Z Z}
\end{array}\right) .
$$

The PT two-point component of the neutral current amplitude for the interaction between fermions with charges $Q, Q^{\prime}$ and isospins $I_{3}, I_{3}^{\prime}$ is then given in terms of the inverse of the matrix $\hat{\Gamma}_{N C}$ by the expression

$$
\begin{aligned}
& \left(\begin{array}{ll}
e Q^{\prime} & \frac{e}{s_{\mathrm{w}} c_{\mathrm{w}}}\left(I_{3}^{\prime}-s_{\mathrm{w}}^{2} Q^{\prime}\right)
\end{array}\right) \hat{\Gamma}_{N C}^{-1}\left(\begin{array}{c}
e Q \\
\frac{e}{s_{\mathrm{w}} c_{\mathrm{w}}}\left(I_{3}-s_{\mathrm{w}}^{2} Q\right)
\end{array}\right)= \\
& \left(\begin{array}{cc}
e Q^{\prime} & \frac{e}{s_{\mathrm{w}} c_{\mathrm{w}}}\left(I_{3}^{\prime}-s_{\mathrm{w}, \mathrm{eff}}^{2}\left(q^{2}\right) Q^{\prime}\right)
\end{array}\right)\left(\begin{array}{cc}
\hat{\Delta}_{\gamma}\left(q^{2}\right) & 0 \\
0 & \hat{\Delta}_{Z}\left(q^{2}\right)
\end{array}\right)\left(\begin{array}{c}
e Q \\
\frac{e}{s_{\mathrm{w}} c_{\mathrm{w}}}\left(I_{3}-s_{\mathrm{w}, \mathrm{eff}}^{2}\left(q^{2}\right) Q\right)
\end{array}\right) .
\end{aligned}
$$

The r.h.s. of this equation, where the neutral current interaction between the fermions has been written in diagonal (i.e. Born-like) form, defines the diagonal propagator functions $\hat{\Delta}_{\gamma}$ and $\hat{\Delta}_{Z}$ and the effective weak mixing angle $s_{\mathrm{w}, \mathrm{eff}}^{2}$. They are given by

$$
\begin{aligned}
\hat{\Delta}_{\gamma}\left(q^{2}\right) & =\frac{1}{q^{2}+\hat{\Sigma}_{\gamma \gamma}\left(q^{2}\right)}, \\
\hat{\Delta}_{Z}\left(q^{2}\right) & =\frac{1}{q^{2}-M_{Z}^{2}+\hat{\Sigma}_{Z Z}\left(q^{2}\right)-\hat{\Sigma}_{\gamma Z}^{2}\left(q^{2}\right) /\left(q^{2}+\hat{\Sigma}_{\gamma \gamma}\left(q^{2}\right)\right)},
\end{aligned}
$$


and

$$
s_{\mathrm{w}, \mathrm{eff}}^{2}\left(q^{2}\right)=s_{\mathrm{w}}^{2}\left(1+\frac{c_{\mathrm{w}}}{s_{\mathrm{w}}} \frac{\hat{\Sigma}_{\gamma Z}\left(q^{2}\right)}{q^{2}+\hat{\Sigma}_{\gamma \gamma}\left(q^{2}\right)}\right)=s_{R, \mathrm{w}}^{2}\left(1+\frac{c_{R, \mathrm{w}}}{s_{R, \mathrm{w}}} \frac{\hat{\Sigma}_{R, \gamma Z}\left(q^{2}\right)}{q^{2}+\hat{\Sigma}_{R, \gamma \gamma}\left(q^{2}\right)}\right) .
$$

In App. B, it is shown how the amplitude (5.2) for the Dyson-summed PT bare two-point component of the neutral current interaction between fermions takes exactly the same form when expressed in terms of renormalized quantities. This is a direct result of the tree-levellike Ward identities obeyed by the PT one-loop $n$-point functions. The effective weak mixing angle $s_{\mathrm{w} \text {,eff }}^{2}$, defined above in terms of bare quantities in the first equality in (5.5), therefore takes exactly the same form when expressed in terms of the corresponding renormalized quantities as in the second equality in (5.5). It is emphasized that the expressions Eqs. (5.3)(5.5) are valid to all orders in perturbation theory (although, to date, the PT proper selfenergies have only been computed to one-loop order).

The amplitude for the Dyson-summed PT two-point component of the charged current interaction between fermions may be written

$$
\frac{e^{2}}{2 s_{\mathrm{w}}^{2}}\left(I_{+} I_{-}^{\prime}+I_{-} I_{+}^{\prime}\right) \hat{\Delta}_{W}\left(q^{2}\right)
$$

where $I_{+}, I_{-}$are the $\mathrm{SU}(2)_{L}$ isospin charge raising and lowering operators, and the propagator function is given by

$$
\hat{\Delta}_{W}\left(q^{2}\right)=\frac{1}{q^{2}-M_{W}^{2}+\hat{\Sigma}_{W W}} .
$$

In App. B, the amplitude (5.6) is also shown to take exactly the same form when expressed in terms of renormalized quantities.

We see that at the one-loop level, the only effect of the $\hat{\Sigma}_{\gamma Z}$ self-energy is to correct the weak mixing angle. At the one-loop level, the complex pole positions $\bar{s}_{\gamma}, \bar{s}_{Z}$ and $\bar{s}_{W}$ of the diagonal propagators $\hat{\Delta}_{\gamma}, \hat{\Delta}_{Z}$ and $\hat{\Delta}_{W}$ are thus given by the solutions of

$$
\bar{s}_{i}-M_{i}^{2}+\hat{\Sigma}_{i i}\left(\bar{s}_{i}\right)=0, \quad i=\gamma, Z, W
$$

(no sum over $i$; for the photon, $M_{\gamma}=0$ and $\hat{\Sigma}_{\gamma \gamma}(0)=0$ so that $\bar{s}_{\gamma}=0$.) The bare one-loop self-energy functions $\hat{\Sigma}_{\gamma \gamma}, \hat{\Sigma}_{Z Z}$ and $\hat{\Sigma}_{W W}$ may be expanded around the corresponding pole positions $\bar{s}_{\gamma}, \bar{s}_{Z}$ and $\bar{s}_{W}$ as

$$
\hat{\Sigma}_{i i}\left(q^{2}\right)=\hat{\Sigma}_{i i}\left(\bar{s}_{i}\right)+\left(q^{2}-\bar{s}_{i}\right) \hat{\Pi}_{i i}\left(q^{2}\right), \quad i=\gamma, Z, W .
$$

The diagonal propagators $\hat{\Delta}_{\gamma}, \hat{\Delta}_{Z}$ and $\hat{\Delta}_{W}$ can then be written in terms of the functions $\hat{\Pi}_{\gamma \gamma}$, $\hat{\Pi}_{Z Z}$ and $\hat{\Pi}_{W W}$ as

$$
\hat{\Delta}_{i}\left(q^{2}\right)=\frac{1}{\left(q^{2}-\bar{s}_{i}\right)\left(1+\hat{\Pi}_{i i}\left(q^{2}\right)\right)}, \quad i=\gamma, Z, W
$$

\footnotetext{
${ }^{\dagger \dagger}$ For a discussion of expansions of the Dyson-summed electroweak self-energies about the pole positions, see Ref. 33.
} 
i.e. with the pole explicitly factored out in each case. The Dyson series of one-loop electroweak radiative corrections included in these propagators may then be fully accounted for by the three effective charges

$$
\begin{aligned}
\alpha_{\mathrm{eff}}\left(q^{2}\right) & =\frac{e^{2}}{4 \pi} \frac{1}{1+\hat{\Pi}_{\gamma \gamma}\left(q^{2}\right)}=\frac{e_{R}^{2}}{4 \pi} \frac{1}{1+\hat{\Pi}_{R, \gamma \gamma}\left(q^{2}\right)}, \\
\alpha_{Z, \mathrm{eff}}\left(q^{2}\right) & =\frac{e^{2}}{4 \pi s_{\mathrm{w}}^{2} c_{\mathrm{w}}^{2}} \frac{1}{1+\hat{\Pi}_{Z Z}\left(q^{2}\right)}=\frac{e_{R}^{2}}{4 \pi s_{R, \mathrm{w}}^{2} c_{R, \mathrm{w}}^{2}} \frac{1}{1+\hat{\Pi}_{R, Z Z}\left(q^{2}\right)}, \\
\alpha_{W, \mathrm{eff}}\left(q^{2}\right) & =\frac{e^{2}}{4 \pi s_{\mathrm{w}}^{2}} \frac{1}{1+\hat{\Pi}_{W W}\left(q^{2}\right)}=\frac{e_{R}^{2}}{4 \pi s_{R, \mathrm{w}}^{2}} \frac{1}{1+\hat{\Pi}_{R, W W}\left(q^{2}\right)} .
\end{aligned}
$$

In Eqs. (5.11)-(5.13), the effective charges have also been written in terms of the corresponding renormalized quantities. Again, this is a direct result of the tree-level-like Ward identities obeyed by the PT one-loop $n$-point functions, as described in App. B. In the on-shell scheme,

the subtraction points used in Eq. (4.1) to define the renormalized self-energy functions are given by the corresponding pole positions. In this scheme, the functions $\hat{\Pi}_{R, i i}$ are then related to the functions $\hat{\Sigma}_{R, i i}\left(q^{2}\right)$ by the simple expressions

$$
\begin{aligned}
\hat{\Sigma}_{R, i i}\left(q^{2}\right) & =\left(q^{2}-\bar{s}_{i}\right)\left(\hat{\Pi}_{i i}\left(q^{2}\right)-\hat{\Pi}_{i i}\left(\bar{s}_{i}\right)\right) \\
& =\left(q^{2}-\bar{s}_{i}\right) \hat{\Pi}_{R, i i}\left(q^{2}\right), \quad i=\gamma, Z, W .
\end{aligned}
$$

Thus, we see that the radiative corrections to the tree level electroweak two-point functions are fully accounted for by four independent effective quantities: the effective weak mixing angle in Eq. (5.5), and the three effective charges in Eqs. (5.11)-(5.13). In an exactly similar way to the QED effective charge in Eq. (2.6), these four electroweak effective quantities are gauge-independent, since the self-energy functions $\hat{\Sigma}_{\gamma Z}, \hat{\Sigma}_{\gamma \gamma}, \hat{\Sigma}_{Z Z}$ and $\hat{\Sigma}_{W W}$ are gaugeindependent; and also manifestly renormalization scale- and scheme-independent, since they may be expressed entirely in terms of bare quantities.

\section{The Effective Charges and the Renormalization Group}

It is instructive to compare the effective charges constructed in the previous section with the corresponding running quantities defined from the renormalization group. In particular, we will show how at high energy the four independent effective quantities $\alpha_{\text {eff }}, \alpha_{Z \text {,eff }}, \alpha_{W \text {,eff }}$ and $s_{\mathrm{w}, \mathrm{eff}}^{2}$ in Eqs. (5.11)-(5.13) and (5.5) may be expressed in terms of just two independent running quantities $\bar{\alpha}$ and $\bar{s}_{\mathrm{w}}^{2}$.

The two $\beta$-functions associated with the electroweak gauge groups $\mathrm{SU}(2)_{L}$ and $\mathrm{U}(1)_{Y}$ are defined in terms of the corresponding renormalized couplings $g_{R}$ and $g_{R}^{\prime}$, respectively, by

$$
\begin{aligned}
\beta\left(g_{R}, g_{R}^{\prime}\right) & =\frac{\mu}{g_{R}^{2}} \frac{d g_{R}^{2}}{d \mu}=\beta_{1} \frac{g_{R}^{2}}{4 \pi^{2}}+\ldots \\
\beta^{\prime}\left(g_{R}, g_{R}^{\prime}\right) & =\frac{\mu}{g_{R}^{\prime 2}} \frac{d g_{R}^{\prime 2}}{d \mu}=\beta_{1}^{\prime} \frac{g_{R}^{\prime 2}}{4 \pi^{2}}+\ldots
\end{aligned}
$$


where $\mu$ is the renormalization scale. The first coefficients in the perturbative expansions are given by

$$
\begin{aligned}
& \beta_{1}=-\frac{43}{12}+\frac{2}{3} N_{g}, \\
& \beta_{1}^{\prime}=+\frac{1}{12}+\frac{10}{9} N_{g},
\end{aligned}
$$

for $N_{g}$ fermion generations. The term $+\frac{1}{12}$ in the expression for $\beta_{1}^{\prime}$ is the contribution of the complex Higgs doublet of scalar fields, while the term $-\frac{43}{12}=-\frac{11}{6} \times 2+\frac{1}{12}$ in the expression for $\beta_{1}$ is the sum of the contributions of the pure $\mathrm{SU}(2)_{L}$ gauge fields and the Higgs doublet. The solutions of the two differential equations in Eqs. (6.1) and (6.2) then define the $\mathrm{SU}(2)_{L}$ and $\mathrm{U}(1)_{Y}$ running couplings $\bar{g}^{2}$ and $\bar{g}^{\prime 2}$, respectively: in the leading-logarithm approximation,

$$
\begin{aligned}
\bar{g}^{2}\left(\mu^{2}\right) & =\frac{g_{R}^{2}}{1-\frac{g_{R}^{2}}{4 \pi^{2}} \beta_{1} \frac{1}{2} \ln \left(\mu^{2} / \mu_{0}^{2}\right)}, \\
\bar{g}^{\prime 2}\left(\mu^{2}\right) & =\frac{g_{R}^{\prime 2}}{1-\frac{g_{R}^{\prime 2}}{4 \pi^{2}} \beta_{1}^{\prime} \frac{1}{2} \ln \left(\mu^{2} / \mu_{0}^{2}\right)},
\end{aligned}
$$

where $\bar{g}^{2}\left(\mu_{0}^{2}\right)=g_{R}^{2}$ and $\bar{g}^{\prime 2}\left(\mu_{0}^{2}\right)=g_{R}^{\prime 2}$.

The two renormalized couplings $g_{R}$ and $g_{R}^{\prime}$ may be traded for a renormalized electromagnetic coupling $\alpha_{R}$ and a renormalized weak mixing angle $s_{R, \mathrm{w}}$, defined by

$$
\begin{aligned}
\alpha_{R} & =\frac{1}{4 \pi} \frac{g_{R}^{2} g_{R}^{\prime 2}}{g_{R}^{2}+g_{R}^{\prime 2}}, \\
s_{R, \mathrm{w}}^{2} & =\frac{g_{R}^{\prime 2}}{g_{R}^{2}+g_{R}^{\prime 2}} .
\end{aligned}
$$

These definitions are by analogy with those of the tree level electromagnetic coupling $\alpha$ and tree level weak mixing angle $s_{\mathrm{w}}$ in terms of the bare couplings $g$ and $g^{\prime}$. The corresponding renormalization group functions $\beta^{\mathrm{em}}$ and $\delta^{s_{\mathrm{w}}}$ associated with $\alpha_{R}$ and $s_{R, \mathrm{w}}$ are then defined by

$$
\begin{aligned}
\beta^{\mathrm{em}}\left(\alpha_{R}, s_{R, \mathrm{w}}\right) & =\frac{\mu}{\alpha_{R}} \frac{d \alpha_{R}}{d \mu}=\beta_{1}^{\mathrm{em}} \frac{\alpha_{R}}{\pi}+\ldots, \\
\delta^{s_{\mathrm{w}}}\left(\alpha_{R}, s_{R, \mathrm{w}}\right) & =-\mu \frac{d s_{R, \mathrm{w}}^{2}}{d \mu}=\delta_{1}^{s_{\mathrm{w}}} \frac{\alpha_{R}}{\pi}+\ldots,
\end{aligned}
$$

(the minus sign in Eq. (6.10) is for convenience). The function $\beta^{\mathrm{em}}$ is thus the $\beta$-function for the electromagnetic coupling in the presence of the electroweak interactions. From the definitions Eqs. (6.1), (6.2) and (6.7), (6.8), the functions $\beta^{\mathrm{em}}$ and $\delta^{s_{\mathrm{w}}}$ are given in terms of $\beta$ and $\beta^{\prime}$ by

$$
\begin{aligned}
\beta^{\mathrm{em}} & =\beta s_{R, \mathrm{w}}^{2}+\beta^{\prime} c_{R, \mathrm{w}}^{2}, \\
\delta^{s_{\mathrm{w}}} & =\left(\beta-\beta^{\prime}\right) s_{R, \mathrm{w}}^{2} c_{R, \mathrm{w}}^{2}
\end{aligned}
$$


where $c_{R, \mathrm{w}}^{2}=1-s_{R, \mathrm{w}}^{2}$. The first coefficients in the perturbative expansions of $\beta^{\mathrm{em}}$ and $\delta^{s_{\mathrm{w}}}$ are thus given by

$$
\begin{array}{ll}
\beta_{1}^{\mathrm{em}}=\beta_{1}+\beta_{1}^{\prime} & =-\frac{7}{2}+\frac{16}{9} N_{g}, \\
\delta_{1}^{s_{\mathrm{w}}}=\beta_{1}-s_{R, \mathrm{w}}^{2}\left(\beta_{1}+\beta_{1}^{\prime}\right) & =-\frac{43}{12}+\frac{2}{3} N_{g}-s_{R, \mathrm{w}}^{2}\left(-\frac{7}{2}+\frac{16}{9} N_{g}\right) .
\end{array}
$$

The bosonic contributions to $\beta_{1}^{\mathrm{em}}$ and $\delta_{1}^{s_{\mathrm{w}}}$ are precisely the coefficients which appear in the $e^{+} e^{-} \rightarrow W^{+} W^{-}$tree level cross section components $\hat{\sigma}_{\gamma \gamma}$ and $\hat{\sigma}_{\gamma Z}$, respectively, in the high energy limit $s / M_{W}^{2} \rightarrow \infty$ [cf. Eqs. (3.24) and (3.25), and the third remark which follows them] and hence which appear in the corresponding $W^{+} W^{-}$contributions to the one loop self-energies $\hat{\Sigma}_{\gamma \gamma}$ and $\hat{\Sigma}_{\gamma Z}$ [cf. Eqs. (4.7) and (4.8)]. The running electromagnetic coupling $\bar{\alpha}$ and the running weak mixing angle $\bar{s}_{\mathrm{w}}^{2}$ are then defined as the solutions of the differential equations in Eqs. 6.9) and (6.10) or, equivalently, directly from the running couplings $\bar{g}^{2}$ and $\bar{g}^{\prime 2}$ :

$$
\begin{aligned}
\bar{\alpha}\left(\mu^{2}\right) & =\frac{1}{4 \pi} \frac{\bar{g}^{2}\left(\mu^{2}\right) \bar{g}^{\prime 2}\left(\mu^{2}\right)}{\bar{g}^{2}\left(\mu^{2}\right)+\bar{g}^{\prime 2}\left(\mu^{2}\right)}=\frac{\alpha_{R}}{1-\frac{\alpha_{R}}{\pi} \beta_{1}^{\mathrm{em}} \frac{1}{2} \ln \left(\mu^{2} / \mu_{0}^{2}\right)}, \\
\bar{s}_{\mathrm{w}}^{2}\left(\mu^{2}\right) & =\frac{\bar{g}^{\prime 2}\left(\mu^{2}\right)}{\bar{g}^{2}\left(\mu^{2}\right)+\bar{g}^{\prime 2}\left(\mu^{2}\right)}=s_{R, \mathrm{w}}^{2}-\frac{\frac{\alpha_{R}}{\pi} \delta_{1}^{s_{\mathrm{w}}} \frac{1}{2} \ln \left(\mu^{2} / \mu_{0}^{2}\right)}{1-\frac{\alpha_{R}}{\pi} \beta_{1}^{\mathrm{em}} \frac{1}{2} \ln \left(\mu^{2} / \mu_{0}^{2}\right)}
\end{aligned}
$$

where the explicit expressions for $\bar{\alpha}$ and $\bar{s}_{\mathrm{w}}^{2}$ are again in the leading-logarithm approximation, with $\bar{\alpha}\left(\mu_{0}^{2}\right)=\alpha_{R}$ and $\bar{s}_{\mathrm{w}}^{2}\left(\mu_{0}^{2}\right)=s_{R, \mathrm{w}}^{2}$.

From the definitions Eqs. (5.11)-(5.13), and Eq. (5.5), together with the explicit expressions Eqs. (4.7)-(4.9) and Eqs. (A.8)-(A.12) for the electroweak gauge field contributions to the self-energies, and also the standard fermion and Higgs contributions (not considered here), we obtain:

$$
\begin{aligned}
\lim _{-q^{2} / M_{W}^{2} \rightarrow \infty} \alpha_{\mathrm{eff}}\left(q^{2}\right) & =\bar{\alpha}\left(q^{2}\right), \\
\lim _{-q^{2} / M_{W}^{2} \rightarrow \infty} \alpha_{Z, \mathrm{eff}}\left(q^{2}\right) & =\frac{\bar{\alpha}\left(q^{2}\right)}{\bar{s}_{\mathrm{w}}^{2}\left(q^{2}\right) \bar{c}_{\mathrm{w}}^{2}\left(q^{2}\right)}, \\
\lim _{-q^{2} / M_{W}^{2} \rightarrow \infty} \alpha_{W, \mathrm{eff}}\left(q^{2}\right) & =\frac{\bar{\alpha}\left(q^{2}\right)}{\bar{s}_{\mathrm{w}}^{2}\left(q^{2}\right)}, \\
\lim _{-q^{2} / M_{W}^{2} \rightarrow \infty} s_{\mathrm{w}, \mathrm{eff}}^{2}\left(q^{2}\right) & =\bar{s}_{\mathrm{w}}^{2}\left(q^{2}\right) .
\end{aligned}
$$

Thus, in the high energy limit, when all masses can be neglected, the $q^{2}$-dependence of the four effective quantities $\alpha_{\text {eff }}, \alpha_{Z \text {,eff }}, \alpha_{W \text {,eff }}$ and $s_{\mathrm{w} \text {,eff }}^{2}$ is fully specified by the renormalization group running of the two independent quantities $\bar{\alpha}$ and $\bar{s}_{\mathrm{w}}^{2}$. 


\section{The Effective Charges and the Background Field Method}

In this section we compare the effective charges constructed in Sec. 5 with those obtained directly from one-loop perturbation theory in the background field method (BFM). This comparison is pertinent for two reasons. First, when a non-abelian theory is quantized in the BFM, the background gauge boson self-energies capture fully the renormalization group running of the gauge couplings, just as in QED. The BFM then naturally provides a welldefined field theoretic framework in which the renormalization group running couplings may be extended into the non-asymptotic region to define sets of background field effective charges. Second, as is now well known, there exists a connection between the one-loop PT $n$-point functions and those obtained in the BFM. In Secs. 3 and 4, it was shown that when the QED relation between one-loop contributions to the gauge field self-energy and the tree level cross sections for the corresponding physical processes is extended to the electroweak theory, the gauge field contributions to the electroweak self-energies which result are identical to those obtained in the PT. It is therefore instructive to reconsider the connection between the PT and the BFM in the context of the approach followed here.

In the BFM [27], the bosonic fields are decomposed into background and quantum components, and the gauge fixing for the quantum gauge fields then chosen such that the effective action remains explicitly invariant under gauge transformations of the background fields. As a result of this exact background gauge invariance, the one-particle-irreducible background field $n$-point functions, although still dependent on the quantum gauge fixing parameter $\xi_{Q}$, obey simple QED-like Ward identities to all orders in perturbation theory. In particular, in the BFM formulation of the electroweak theory [28], the renormalization constants for the background $\gamma, Z$ and $W$ gauge fields are $\xi_{Q}$-independent, and are related to those of the electromagnetic coupling and the weak mixing angle precisely as in Eqs. (B.8). Consequently, the set of effective charges defined by the Dyson summation of the background gauge field self-energies are renormalization scale- and scheme-independent, and at asymptotic energies match on to the corresponding running quantities defined from the renormalization group. However, in the non-asymptotic region the BFM effective charges retain a dependence on the quantum gauge parameter $\xi_{Q}$. Thus, the effective charges defined via the Dyson summation of the BFM self-energies, although well-defined at the field theoretic level and while satisfying the constraints of the renormalization group, are not unique.

It has been observed by various authors [28 30] that the PT gauge-independent oneloop $n$-point functions coincide with the background field $n$-point functions computed in the Feynman quantum gauge $\xi_{Q}=1$, both in QCD and the electroweak Standard Model.. Furthermore, it has been argued [28, 32] that the PT $n$-point functions are not distinguished on physical grounds from the BFM $n$-point functions computed for an arbitrary value of

* For an extensive discussion of the connection between the PT and the BFM in perturbation theory at the one-loop level and beyond, see Ref. [31. 
$\xi_{Q}$. In the case of the gauge boson self-energies, this argument leads to the conclusion that, away from the asymptotic region governed by the renormalization group, there is no unique, physically distinguished way in which to extend the concept of an effective charge from QED to the electroweak sector of the Standard Model in particular, and to non-abelian gauge theories in general.

However, as emphasized in Ref. []], for $\xi_{Q} \neq 1$ the imaginary parts of the BFM electroweak self-energies $\Sigma_{i j}^{\mathrm{BFM}}, i, j=\gamma, Z, W$, include terms with unphysical thresholds. For example, for the one-loop contributions of the $W$ and its associated would-be Goldstone boson and ghost to $\Sigma_{Z Z}^{\mathrm{BFM}}$ [32], one obtains

$$
\begin{aligned}
\operatorname{Im} & \Sigma_{Z Z}^{\mathrm{BFM}(W W}\left(\xi_{Q}, q^{2}\right)=\operatorname{Im} \hat{\Sigma}_{Z Z}^{(W W)}\left(q^{2}\right)+\frac{\alpha}{24 s_{\mathrm{w}}^{2} c_{\mathrm{w}}^{2}}\left(q^{2}-M_{Z}^{2}\right) \frac{1}{M_{Z}^{4}} \times \\
& \left\{\left(\left(8 M_{W}^{2}+q^{2}\right)\left(M_{Z}^{2}+q^{2}\right)+4 M_{W}^{2}\left(4 M_{W}^{2}+3 M_{Z}^{2}+2 q^{2}\right)\right) \lambda^{\frac{1}{2}}\left(q^{2}, M_{W}, M_{W}\right) \vartheta\left(q^{2}-4 M_{W}^{2}\right)\right. \\
& +\left(\left(8 M_{W}^{2}+q^{2}\right)\left(M_{Z}^{2}+q^{2}\right)-4 M_{W}^{2}\left(4 M_{W}^{2}+3 M_{Z}^{2}+2 q^{2}\right)\right. \\
& \left.-4\left(\xi_{Q}-1\right) M_{W}^{2}\left(4 M_{W}^{2}+M_{Z}^{2}+q^{2}\right)\right) \lambda^{\frac{1}{2}}\left(q^{2}, \sqrt{\xi}_{Q} M_{W}, \sqrt{\xi}_{Q} M_{W}\right) \vartheta\left(q^{2}-4 \xi_{Q} M_{W}^{2}\right) \\
& -2\left(8 M_{W}^{2}+q^{2}-2\left(\xi_{Q}-1\right) M_{W}^{2}+\left(\xi_{Q}-1\right)^{2} M_{W}^{4} q^{-2}\right)\left(M_{Z}^{2}+q^{2}\right) \times \\
& \left.\lambda^{\frac{1}{2}}\left(q^{2}, M_{W}, \sqrt{\xi_{Q}} M_{W}\right) \vartheta\left(q^{2}-\left(1+\sqrt{\xi}_{Q}\right)^{2} M_{W}^{2}\right)\right\},
\end{aligned}
$$

where $\lambda\left(q^{2}, M_{i}, M_{j}\right)$ was defined after Eq. (3.30). These gauge-dependent unphysical thresholds are artefacts of the BFM electroweak $R_{\xi}$-like gauge fixing procedure, and exactly cancel in the calculation of any physical process. For example, one can consider in the framework of the BFM the radiative corrections to the process $e^{+} e^{-} \rightarrow e^{+} e^{-}$in the case of forward scattering, as discussed in the introduction. The contributions of the $W$ and its associated would-be Goldstone boson and ghost to the one-loop BFM radiative corrections are just as illustrated schematically on the 1.h.s. of Fig. 3. The unphysical thresholds which occur in the imaginary parts of the various one-loop self-energy contributions are then exactly cancelled by corresponding unphysical contributions from the imaginary parts of the one-loop vertex and box contributions. Thus, as stated in the introduction, these cancellations leave just the contribution proportional to the tree level cross section for the on-shell physical process $e^{+} e^{-} \rightarrow W^{+} W^{-}$, illustrated on the r.h.s. of Fig. 3, with threshold at $q^{2}=4 M_{W}^{2}$.

Clearly, the unphysical thresholds which occur in the imaginary parts of the electroweak $n$-point functions in the BFM (or indeed with any other gauge fixing procedure) are not experimentally measurable quantities. It follows that the gauge field contributions to the renormalized BFM electroweak self-energies $\Sigma_{R, i j}^{\mathrm{BFM}}\left(\xi_{Q}, q^{2}\right)$ computed with $\xi_{Q} \neq 1$ cannot be reconstructed from the tree level cross sections for the corresponding on-shell physical processes. Furthermore, as remarked in Ref. [32] (third paper), it is not a priori obvious that, even for $\xi_{Q}=1$, all thresholds in the imaginary parts of the BFM self-energies are due to physical fields. Here, by obtaining the full one-loop $W$ contribution to the renormalized PT 
neutral current self-energies, coincident with the BFM self-energies at $\xi_{Q}=1$, directly from the tree level cross section for the on-shell physical process $e^{+} e^{-} \rightarrow W^{+} W^{-}$, we have shown explicitly that, in the BFM at $\xi_{Q}=1$, the thresholds which occur at $q^{2}=4 M_{W}^{2}$ are due solely to the physical $W^{+} W^{-}$pair. We therefore conclude that the particular value $\xi_{Q}=1$ in the BFM precisely is distinguished on physical grounds from all other values of $\xi_{Q}$.

\section{Phenomenological Determination of the Effective Charges}

Finally, we turn to the extraction from experiment of the effective charges and weak mixing angle. In QED, the fine structure constant $\alpha$ together with the mass(es) of the fermion(s) provides the experimental input required to determine the parameters of the theory. The overall scale of the effective charge is then determined by $\alpha$, while in the on-shell scheme the subtraction point in the dispersion relation Eq. (2.4) for the renormalized vacuum polarization function is at $s=0$. The one-loop contributions to the spectral function $\operatorname{Im} \Pi$ are then directly proportional to components of the tree level cross sections for the corresponding fermion scattering processes, as described in the Introduction.

In the electroweak Standard Model, the parameters of the theory are determined by three independent experimental inputs together with the masses of the fermions and the Higgs boson. In the on-shell scheme, these three parameters are chosen to be the fine structure constant, defined from Compton scattering in the classical Thomson limit, and the masses of the $W$ and $Z$ gauge bosons, defined from the pole positions of the corresponding propagators.

At the one-loop level in the electroweak theory, the quantum correction to the fine structure constant which appears in the classical Compton scattering process is given by

$$
\begin{aligned}
\alpha & =\frac{e^{2}}{4 \pi}\left(1-\Pi_{\gamma \gamma}(\xi, 0)-\frac{2 s_{\mathrm{w}}}{c_{\mathrm{w}}} \frac{\Sigma_{\gamma Z}(\xi, 0)}{M_{Z}^{2}}\right) \\
& =\frac{e^{2}}{4 \pi}\left(1-\hat{\Pi}_{\gamma \gamma}(0)\right)=\frac{e_{R}^{2}}{4 \pi}\left(1-\hat{\Pi}_{R, \gamma \gamma}(0)\right) .
\end{aligned}
$$

In Eq. (8.1), $\Pi_{\gamma \gamma}\left(\xi, q^{2}\right)$ and $\Sigma_{\gamma Z}\left(\xi, q^{2}\right)$ are the conventional gauge-dependent photon vacuum polarization and $\gamma-Z$ self-energy in the class of renormalizable $\left(R_{\xi}\right)$ gauges with (common) gauge parameter $\xi$. At vanishing four-momentum transfer $q^{2}=0$, the combination of these two functions in Eq. (8.1) is gauge-independent and is precisely equal to the the PT function $\hat{\Pi}_{\gamma \gamma}\left(q^{2}\right)$ at $q^{2}=0$. $\mathrm{f}$ Thus, the PT photon vacuum polarization specifies fully the one-loop electroweak radiative corrections to the classical Compton scattering process in the Thomson limit. This is precisely analogous to QED. In the second equality in (8.2), the Ward identity

\footnotetext{
${ }^{\dagger}$ For a discussion of the pathologies which result from the Dyson summation of self-energies which include unphysical thresholds, see Ref [7].

$\ddagger$ The expressions for the PT functions $\hat{\Pi}_{\gamma \gamma}\left(q^{2}\right)$ and $\hat{\Sigma}_{\gamma Z}\left(q^{2}\right)$ in terms of $\Pi_{\gamma \gamma}\left(\xi=1, q^{2}\right)$ and $\Sigma_{\gamma Z}\left(\xi=1, q^{2}\right)$ can be found in Eqs. (16a) and (16b) of Ref. [13. Using these expressions, together with the fact that $\hat{\Sigma}_{\gamma Z}(0)=0$, one obtains the first equality in (8.2).
} 
Eq. (B.10) has been used to write the one-loop expression for $\alpha$ in terms of renormalized quantities. In the on-shell scheme, with subtraction point $s_{\gamma \gamma}=0, \hat{\Pi}_{R, \gamma \gamma}(0)=0$ and $e_{R}$ is identified with the physical electron charge.

Also at the one-loop level, the pole positions $\bar{s}_{Z}$ and $\bar{s}_{W}$ of the conventional $R_{\xi}$ gauge $Z$ and $W$ propagators are given by

$$
\begin{aligned}
\bar{s}_{i} & =M_{i}^{2}-\Sigma_{i i}\left(\xi, M_{i}^{2}\right) \\
& =M_{i}^{2}-\hat{\Sigma}_{i i}\left(M_{i}^{2}\right)=M_{R, i}^{2}+\delta M_{i}^{2}-\hat{\Sigma}_{i i}\left(M_{i}^{2}\right), \quad i=Z, W .
\end{aligned}
$$

At $q^{2}=M_{Z}^{2}\left[M_{W}^{2}\right]$, the conventional $R_{\xi}$ gauge self-energy function $\Sigma_{Z Z}\left(\xi, q^{2}\right)\left[\Sigma_{W W}\left(\xi, q^{2}\right)\right]$ is gauge-independent and is precisely equal to the PT function $\hat{\Sigma}_{Z Z}\left(q^{2}\right)\left[\hat{\Sigma}_{W W}\left(q^{2}\right)\right]$ at $q^{2}=M_{Z}^{2}$ $\left[M_{W}^{2}\right]$. Thus, the PT self-energies do not shift the position of the complex poles. This was shown to one-loop order in Refs. [12, 13] and to higher orders in Ref. [6]. In the second equality in (8.4), the bare masses have been written in terms of renormalized masses and counterterms. In the on-shell scheme, with renormalization conditions $\delta M_{i}^{2}=\operatorname{Re} \hat{\Sigma}_{i i}\left(M_{i}^{2}\right)$, $i=Z, W, M_{R, Z}$ and $M_{R, W}$ are identified with the physical $Z$ and $W$ masses. In this scheme, the renormalized weak mixing angle is then defined by

$$
c_{R, \mathrm{w}}^{2}=1-s_{R, \mathrm{w}}^{2}=\frac{M_{R, W}^{2}}{M_{R, Z}^{2}} .
$$

Thus, in the on-shell scheme at the one-loop level, the overall scale of each of the three effective charges Eqs. (5.11)-(5.13) and the effective weak mixing angle Eq. (5.5) is given by the fine structure constant $\alpha$ and the ratio $c_{R, \mathrm{w}}$ of the physical $W$ and $Z$ masses; the subtraction points $s_{\gamma \gamma}$ and $s_{\gamma Z}$ for $\hat{\Sigma}_{R, \gamma \gamma}$ and $\hat{\Sigma}_{R, \gamma Z}$ in the dispersion relation Eq. (4.2) are both at zero; and the subtraction points $s_{Z Z}$ and $s_{W W}$ for $\hat{\Sigma}_{R, Z Z}$ and $\hat{\Sigma}_{R, W W}$ in the dispersion relation Eq. (4.2) are given by the squares of the physical $Z$ and $W$ masses, respectively.

It remains to extract from experiment the absorptive parts $\operatorname{Im} \hat{\Sigma}_{\gamma \gamma}, \operatorname{Im} \hat{\Sigma}_{\gamma Z}, \operatorname{Im} \hat{\Sigma}_{Z Z}$ and $\operatorname{Im} \hat{\Sigma}_{W W}$ of the electroweak self-energy functions. In QED, the one-loop muon contribution of to the spectral function Im $\Pi$ is determined directly from the tree level cross section $\sigma\left(e^{+} e^{-} \rightarrow\right.$ $\left.\mu^{+} \mu^{-}\right)$. For the one-loop electron contribution to $\operatorname{Im} \Pi$, however, it is necesssary to project out the self-energy-like component of the tree level cross section $\sigma\left(e^{+} e^{-} \rightarrow e^{+} e^{-}\right)$. This is possible due to the linear independence of the self-energy-, vertex- and box-like components of the Bhabha differential cross section Eq. (2.1). Similarly, in the electroweak Standard Model, in order to obtain the gauge boson contributions to the functions $\operatorname{Im} \hat{\Sigma}_{\gamma \gamma}, \operatorname{Im} \hat{\Sigma}_{\gamma Z}$, $\operatorname{Im} \hat{\Sigma}_{Z Z}$ and $\operatorname{Im} \hat{\Sigma}_{W W}$, it is necessary to project out the self-energy-like components of the physical cross sections $e^{+} e^{-} \rightarrow W^{+} W^{-}$and $e^{+} e^{-} \rightarrow Z H$ for the neutral current functions, and $e^{+} \nu_{e} \rightarrow W^{+} Z, e^{+} \nu_{e} \rightarrow W^{+} \gamma$ and $e^{+} \nu_{e} \rightarrow W^{+} H$ for the charged current function. These projections can be obtained by the appropriate convolution of the full differential cross sections with specific angular functions. To illustrate the procedure, we shall explicitly discuss the simplified limit in which the weak mixing angle is set to zero, i.e. the case of a broken $\mathrm{SU}(2)_{L}$ 
gauge theory. In this case, it is sufficient to know the differential cross sections in order to make the projection. The general case with $s_{\mathrm{w}}^{2} \neq 0$ requires in addition the observation of spin density matrices [34]; though technically more involved, the procedure is in principle the same. The important property which we wish to stress is that, in contrast to the conventional gauge-dependent self-energies, the absorptive parts $\operatorname{Im} \hat{\Sigma}_{\gamma \gamma}, \operatorname{Im} \hat{\Sigma}_{\gamma Z}, \operatorname{Im} \hat{\Sigma}_{Z Z}$ and $\operatorname{Im} \hat{\Sigma}_{W W}$ of the pinch technique self-energies are directly related to components of physical cross sections which are, in principle, experimentally observable.

We thus set $s_{\mathrm{w}}^{2}=0$ in Eqs. (3.18)-(3.23), with $\alpha=g^{2} s_{\mathrm{w}}^{2} / 4 \pi$. It is also convenient to introduce the variables

$$
x=\cos \theta, \quad z=\frac{1+\beta^{2}}{2 \beta} .
$$

Then

$$
\frac{s}{t}=-\frac{2}{\beta} \frac{1}{(z-x)} .
$$

The function $\hat{M}_{\nu \nu}$ Eq. (3.23) specifying the box-like contribution to the differential cross section has a double pole at the (unphysical) point $z=x$; the function $\hat{M}_{Z \nu}$ Eq. (3.22) specifying the vertex-like contribution has a single pole at $z=x$; and the function $\hat{M}_{Z Z}$ Eq. (3.20) specifying the self-energy-like contribution has no pole. If the differential cross section is multiplied by the factor $(z-x)^{2}$, the resulting observable then has a simple degree four polynomial dependence on the variable $x=\cos \theta$. The product of the differential cross section and $(z-x)^{2}$ can therefore be expanded in terms of any set of five linearly independent polynomials $F_{i}(s, x), i=1,2 \ldots 5$ of degree four in $x$, and which may also depend on $s$ :

$$
\left.(z-x)^{2} \frac{d \sigma\left(e^{+} e^{-} \rightarrow W^{+} W^{-}\right)}{d x}\right|_{s_{\mathrm{w}}=0}=\frac{g^{4}}{64 \pi} \beta \frac{s}{\left(s-M^{2}\right)^{2}} \sum_{i=1}^{5} A_{i}(s) F_{i}(s, x)
$$

(we have used $d \Omega=2 \pi d x$ and on the r.h.s. extracted an overall factor for convenience).

We now make the following choice for the polynomials $F_{i}(s, x)$, with from Eqs. (3.8), (3.20), (3.22) and (3.23) the corresponding coefficient functions $A_{i}(s)$ :

$$
\begin{array}{ll}
F_{1}(s, x)=(z-x)^{2} & A_{1}(s)=\frac{5}{32}\left(\beta^{2}-12\right) \vartheta\left(s-4 M^{2}\right), \\
F_{2}(s, x)=(z-x)^{2} x^{2} & A_{2}(s)=-\frac{9}{32} \beta^{2} \vartheta\left(s-4 M^{2}\right) ; \\
F_{3}(s, x)=(z-x)\left(1-x^{2}\right) & A_{3}(s)=-\frac{\beta}{2}\left(\frac{s-M^{2}}{s}\right) \vartheta\left(s-4 M^{2}\right), \\
F_{4}(s, x)=(z-x)(1-\beta x) & A_{4}(s)=\frac{2}{\beta}\left(\frac{s-M^{2}}{s}\right) \vartheta\left(s-4 M^{2}\right) ; \\
F_{5}(s, x)=1-x^{2} & A_{5}(s)=\frac{1}{2}\left(\frac{s-M^{2}}{s}\right)^{2} \vartheta\left(s-4 M^{2}\right) .
\end{array}
$$

The above choice of polynomials $F_{i}(s, x)$ is such as to isolate explicitly the self-energy-like, vertex-like and box-like components of the differential cross section. Thus, the coefficients 
$A_{1}(s)$ and $A_{2}(s)$ contribute only to the self-energy-like component $d \hat{\sigma}_{Z Z} / d x$; the coefficients $A_{3}(s)$ and $A_{4}(s)$ contribute only to the vertex-like component $d \hat{\sigma}_{Z \nu} / d x$; and the coefficient $A_{5}(s)$ contributes only to the box-like component $d \hat{\sigma}_{\nu \nu} / d x$. In particular, the imaginary part of the $W$ contribution to the self-energy is then given by

$$
\left.\operatorname{Im} \hat{\Sigma}_{R, Z Z}^{(W W)}(s)\right|_{s_{\mathrm{w}}=0}=\frac{g^{2}}{4 \pi} \beta s\left(A_{1}(s)+\frac{1}{3} A_{2}(s)\right) .
$$

The Wronskian for the five functions $F_{i}(s, x)$ is given by $W\left(F_{i}\right)=288(1-z \beta)\left(1-z^{2}\right)$. The functions are therefore linearly independent for all values of $s$ except at the zeros of $W\left(F_{i}\right)$ occurring at $z=\beta^{-1}$ and (equivalently) $z=1$, i.e. at $s / M_{W}^{2} \rightarrow \infty$.

To project out the functions $A_{i}(s)$ we construct a further set of five degree four polynomials $\tilde{F}_{i}(s, x)$ satisfying the orthogonality conditions

$$
\int_{-1}^{1} d x F_{i}(s, x) \tilde{F}_{j}(s, x)=\delta_{i j}, \quad i, j=1,2 \ldots 5 .
$$

The explicit expressions for the $\tilde{F}_{i}(s, x)$ corresponding to the specific choice of $F_{i}(s, x)$ in (8.9) are given in App. C. Using these functions $\tilde{F}_{i}(s, x)$, the coefficient functions $A_{i}(s)$ may then be projected out from the observable formed from the product of the differential cross section and the kinematic factor $(z-x)^{2}$ :

$$
\left.\int_{-1}^{1} d x \tilde{F}_{i}(s, x)(z-x)^{2} \frac{d \sigma}{d x}\right|_{s_{\mathrm{w}}=0}=\frac{g^{4}}{64 \pi} \beta \frac{s}{\left(s-M^{2}\right)^{2}} A_{i}(s) .
$$

Thus, by expanding the product of the differential cross section and $(z-x)^{2}$ in terms of functions $F_{i}(s, x)$ which characterize explicitly the angular $(x)$ dependence of the self-energy-, vertex- and box-like components of the differential cross section, it is possible to extract $\left.\operatorname{Im} \hat{\Sigma}_{R, Z Z}^{(W W)}(s)\right|_{s_{\mathrm{w}}=0}$ directly from $d \sigma\left(e^{+} e^{-} \rightarrow W^{+} W^{-}\right) /\left.d x\right|_{s_{\mathrm{w}}=0}$.

\section{Conclusions}

The analysis presented here demonstrates the existence of effective charges in non-abelian gauge theories with properties precisely analogous to those of the well-known effective charge of QED. We have shown this by an explicit construction in the neutral current sector of the electroweak Standard Model, where the processes $e^{+} e^{-} \rightarrow W^{+} W^{-}$and $e^{+} e^{-} \rightarrow Z H$ play the same rôle as the processes $e^{+} e^{-} \rightarrow f^{+} f^{-}$in QED with fermions $f=e, \mu \ldots$.

For the process $e^{+} e^{-} \rightarrow W^{+} W^{-}$, we have shown how the tree level cross section naturally decomposes into components which are uniquely defined according to the propagator structure occurring in the square of the modulus of the S-matrix element, and which are individually well-behaved at high energy. This decomposition follows directly from the systematic use of tree level Ward identities to implement cancellations among contributions originating from diagrams with distinct $s$ - and $t$-dependence. These cancellations are purely non-abelian in 
character, and have no analogue in QED. The resulting expressions for the self-energy-like components of the tree level cross section explicitly display the $W$ contributions to the oneloop electroweak $\beta$-functions, in the same way as the self-energy-like components of the tree level $e^{+} e^{-} \rightarrow f^{+} f^{-}$cross sections display the corresponding fermion contributions. We have then used the self-energy-like components of the tree level $e^{+} e^{-} \rightarrow W^{+} W^{-}$cross section and the full tree level $e^{+} e^{-} \rightarrow Z H$ cross section to obtain one-loop gauge boson contributions to renormalized electroweak self-energy functions using dispersion relations, by direct analogy with QED. The self-energy functions so obtained are identical to the corresponding functions obtained in the pinch technique. These self-energy functions, together with that for the $W$, were then used to construct the electroweak effective charges and the effective weak mixing angle. We have shown how, in the on-shell renormalization scheme, the subtractions points in the dispersion relations and also the overall scale of the effective charges and weak mixing angle are related to the three experimental inputs used to determine the basic parameters of the electroweak theory. Furthermore, we have described how the gauge boson contributions to the absorptive parts of the self-energy functions may be projected out directly from the corresponding tree level physical cross section.

Radiative corrections to two-point functions in the electroweak Standard Model are thus fully accounted for by four independent functions: the three effective charges in Eqs. (5.11)(5.13), and the effective weak mixing angle in Eq. (5.5). These four effective quantities are gauge-independent, and also renormalization scale- and scheme-independent. In the high energy limit, when all masses can be neglected, the $q^{2}$-dependence of these four effective quantities is fully specified by the renormalization group running of two independent quantities, which may be chosen to be the running electromagnetic coupling $\bar{\alpha}$ and the running weak mixing angle $\bar{s}_{\mathrm{w}}^{2}$. At all other momentum scales where masses cannot be neglected, the effective charges we have constructed provide the unique and unambiguous extension of the QED concept of an effective charge to include the contributions of massive gauge bosons. The comparison of these functions with experiment, along the lines discussed in Ref. [35], should provide a natural way to parameterize possible deviations from the Standard Model due to "new physics".

We consider the fact that the self-energy functions which we have constructed from the physical processes $e^{+} e^{-} \rightarrow W^{+} W^{-}$and $e^{+} e^{-} \rightarrow Z H$ turn out to be identical to those obtained by the pinch technique provides a convincing argument in favour of the pinch technique approach to the construction of effective charges. Indeed, it is remarkable that the simple QED unitarity relation among components of the tree level cross section for the interaction of on-shell particles and the imaginary parts of the corresponding one-loop self-energy, vertex and box functions may be extended to non-abelian gauge theories in this way. Furthermore, the cancellation mechanism illustrated in Fig. 5 responsible for the good high energy behaviour of the tree level $e^{+} e^{-} \rightarrow W^{+} W^{-}$cross section is also that responsible for the gauge-independence of the $W$ contributions to the PT self-energies $\hat{\Sigma}_{\gamma \gamma}, \hat{\Sigma}_{\gamma Z}$ and $\hat{\Sigma}_{Z Z}$ ob- 
tained via the rearrangement of one-loop perturbation theory diagrams. Clearly, it would be worthwhile now to extend this construction to the one-particle-irreducible two-loop level.

The electroweak example which we have worked out also gives support to the QCD effective charge obtained by the pinch technique, recently extensively discussed in ref. [8], as the appropriate quantity to be used in renormalon calculus in the one-loop approximation of the $\beta$-function. In a similar way to the electroweak effective charges constructed here, the QCD effective charge constructed in [8] corresponds to a well-defined class of components of Feynman diagrams selected by the tree level Ward identities.

In spite of the progress which has been made in understanding the concept of an effective charge in non-abelian gauge theories, there remain issues which we still would like to clarify:

- One issue is the possible connection with conformal invariance: once the higher orders of perturbation theory are absorbed in the effective charge, one would expect, in the massless limit, conformal invariance to be restored. What are then the corresponding constraints?

- So far we only have a diagrammatic understanding of the construction of an effective charge. Although, as explained in this paper, we are able to relate this construction to physical observables, we still lack a formal understanding, in particular in terms of a path integral formulation. What is the constraint on the path integral which projects the effective charge two-point functions directly?

We hope to come back to these questions in the near future.

It is a pleasure to thank J. Bernabéu, F. Boudjema, R. Coquereaux, M. Hassler, S. Peris, M. Perrottet and A. Pich for useful discussions. NJW acknowledges the financial support of EC HCM grant ERB4001GT933989. 


\section{Appendix A}

In this Appendix, we give the explicit expressions for the renormalized self-energy functions $\hat{\Sigma}_{R, Z Z}^{(Z H)}, \hat{\Sigma}_{R, W W}^{(W H)}, \hat{\Sigma}_{R, W W}^{(W \gamma)}$ and $\hat{\Sigma}_{R, W W}^{(W Z)}$ defined in Sec. 4 . To this end, it is convenient to introduce the function $L_{i j}$ defined by

$$
L_{i j}\left(q^{2}\right)=\frac{1}{4} \lambda_{i j}^{\frac{1}{2}} \ln \rho_{i j}-1+\frac{1}{2} \ln \frac{2 M_{i} M_{j}}{M_{i}^{2}+M_{j}^{2}}+\frac{1}{2} \kappa_{i j} \ln \frac{M_{i}}{M_{j}},
$$

where

$$
\begin{aligned}
\lambda_{i j} & =\left(1-\frac{\left(M_{i}+M_{j}\right)^{2}}{q^{2}}\right)\left(1-\frac{\left(M_{i}-M_{j}\right)^{2}}{q^{2}}\right), \\
\rho_{i j} & =\frac{M_{i}^{2}+M_{j}^{2}-\left(1+\lambda_{i j}^{\frac{1}{2}}\right) q^{2}}{M_{i}^{2}+M_{j}^{2}-\left(1-\lambda_{i j}^{\frac{1}{2}}\right) q^{2}}, \\
\kappa_{i j} & =\frac{M_{i}^{2}-M_{j}^{2}}{q^{2}} .
\end{aligned}
$$

Thus

$$
\begin{aligned}
\operatorname{Im} L_{i j}\left(q^{2}\right) & =-\frac{\pi}{2} \lambda_{i j}^{\frac{1}{2}} \vartheta\left(q^{2}-\left(M_{i}+M_{j}\right)^{2}\right) \\
q^{2} \frac{d L_{i j}\left(q^{2}\right)}{d q^{2}} & =\frac{1}{\lambda_{i j}}\left\{\left(\frac{M_{i}^{2}+M_{j}^{2}}{q^{2}}-\kappa_{i j}^{2}\right)\left(\frac{1}{4} \lambda_{i j}^{\frac{1}{2}} \ln \rho_{i j}-1\right)+\frac{1}{2}\left(1-\kappa_{i j}^{2}\right)\right\}-\frac{1}{4} \kappa_{i j} \ln \frac{M_{i}}{M_{j}} .
\end{aligned}
$$

For the particular case $i=j=W, \lambda_{W W}^{\frac{1}{2}}=\beta, \rho_{W W}=(\beta+1)^{2} /(\beta-1)^{2}, \kappa_{W W}=0$ and $L_{W W}\left(q^{2}\right)=B\left(q^{2}\right)$ [cf. Eq. (4.10)]. Also, for the case $j=\gamma$, i.e. $M_{j}=0$, Eq. (A.1) reduces to

$$
L_{i \gamma}\left(q^{2}\right)=\frac{1}{2}\left(1-\frac{M_{i}^{2}}{q^{2}}\right) \ln \left(1-\frac{q^{2}}{M_{i}^{2}}\right)+\frac{1}{2} \ln 2-1 .
$$

The $Z H$ and $W H$ one-loop contributions to the self-energies $\hat{\Sigma}_{R, Z Z}$ and $\hat{\Sigma}_{R, W W}$, respectively, renormalized in the on-shell scheme are then given by

$$
\begin{aligned}
\hat{\Sigma}_{R, Z Z}^{(Z H)}\left(q^{2}\right) & =\left.\frac{1}{s_{\mathrm{w}}^{2} c_{\mathrm{w}}^{2}} \hat{\Sigma}_{R, i i}^{(i H)}\left(q^{2}\right)\right|_{i=Z}, \\
\hat{\Sigma}_{R, W W}^{(W H)}\left(q^{2}\right) & =\left.\frac{1}{s_{\mathrm{w}}^{2}} \hat{\Sigma}_{R, i i}^{(i H)}\left(q^{2}\right)\right|_{i=W},
\end{aligned}
$$

where

$$
\begin{aligned}
\hat{\Sigma}_{R, i i}^{(i H)}\left(q^{2}\right)= & \frac{\alpha}{\pi}\left(q^{2}-M_{i}^{2}\right) \times \\
& \left\{\left(-\frac{1}{24}-\frac{5 M_{i}^{2}}{12 q^{2}}+\frac{M_{H}^{2}}{12 q^{2}}-\frac{\left(M_{i}^{2}-M_{H}^{2}\right)^{2}}{24 q^{4}}\right) q^{2}\left(\frac{L_{i H}\left(q^{2}\right)-L_{i H}\left(M_{i}^{2}\right)}{q^{2}-M_{i}^{2}}\right)\right. \\
& -\left.\left(-\frac{1}{2}+\frac{M_{H}^{2}}{6 M_{i}^{2}}-\frac{M_{H}^{4}}{24 M_{i}^{4}}\right) M_{i}^{2} \frac{d L_{i H}\left(q^{2}\right)}{d q^{2}}\right|_{M_{i}^{2}} \\
& \left.-\frac{1}{48}\left(1-\ln \frac{2 M_{i}^{2}}{M_{i}^{2}+M_{H}^{2}}-\frac{M_{H}^{2}}{M_{i}^{2}}\left[1-\ln \frac{2 M_{i}^{2}}{M_{i}^{2}+M_{H}^{2}}\right]\right)\left(1-\frac{M_{H}^{2}}{M_{i}^{2}}\right)\left(1-\frac{M_{i}^{2}}{q^{2}}\right)\right\} .
\end{aligned}
$$


Similarly, the $W \gamma$ and $W Z$ one-loop contributions to the self-energy $\hat{\Sigma}_{R, W W}$ renormalized in the on-shell scheme are given by

$$
\begin{aligned}
\hat{\Sigma}_{R, W W}^{(W \gamma)}\left(q^{2}\right)= & \frac{\alpha}{\pi}\left(q^{2}-M_{W}^{2}\right)\left\{\left(\frac{11}{3}-\frac{4 M_{W}^{2}}{3 q^{2}}-\frac{M_{W}^{4}}{3 q^{4}}\right) q^{2}\left(\frac{L_{W \gamma}\left(q^{2}\right)-L_{W \gamma}\left(M_{W}^{2}\right)}{q^{2}-M_{W}^{2}}\right)\right. \\
& \left.-\left.2 M_{W}^{2} \frac{d L_{W \gamma}\left(q^{2}\right)}{d q^{2}}\right|_{M_{W}^{2}}-\frac{1}{6}(1-\ln 2)\left(1-\frac{M_{W}^{2}}{q^{2}}\right)\right\}, \\
\hat{\Sigma}_{R, W W}^{(W Z)}\left(q^{2}\right)= & \frac{\alpha}{\pi} \frac{c_{\mathrm{w}}^{2}}{s_{\mathrm{w}}^{2}}\left(q^{2}-M_{W}^{2}\right)\left\{\left(\frac{11}{3}-\frac{4 M_{W}^{2}}{3 q^{2}}+\frac{2 M_{Z}^{2}}{3 q^{2}}-\frac{\left(M_{W}^{2}-M_{Z}^{2}\right)^{2}}{3 q^{4}}\right.\right. \\
& \left.+\frac{1}{c_{\mathrm{w}}^{2}}\left[-\frac{1}{24}+\frac{19 M_{W}^{2}}{12 q^{2}}-\frac{5 M_{Z}^{2}}{12 q^{2}}-\frac{\left(M_{W}^{2}-M_{Z}^{2}\right)^{2}}{24 q^{4}}\right]\right) q^{2}\left(\frac{L_{W Z}\left(q^{2}\right)-L_{W Z}\left(M_{W}^{2}\right)}{q^{2}-M_{W}^{2}}\right) \\
& -\left.\left(2+\frac{17}{6 c_{\mathrm{w}}^{2}}-\frac{2}{3 c_{\mathrm{w}}^{4}}-\frac{1}{24 c_{\mathrm{w}}^{6}}\right) M_{W}^{2} \frac{d L_{W Z}\left(q^{2}\right)}{d q^{2}}\right|_{M_{W}^{2}} \\
& \left.-\left(\frac{1}{6}+\frac{1}{48 c_{\mathrm{w}}^{2}}\right)\left(\frac{s_{\mathrm{w}}^{2}}{c_{\mathrm{w}}^{2}} \ln \frac{2}{1+c_{\mathrm{w}}^{2}}+\ln c_{\mathrm{w}}^{2}\right) \frac{s_{\mathrm{w}}^{2}}{c_{\mathrm{w}}^{2}}\left(1-\frac{M_{W}^{2}}{q^{2}}\right)\right\} .
\end{aligned}
$$

Note that in the limit $s_{\mathrm{w}}^{2}=0, \hat{\Sigma}_{R, W W}^{(W \gamma)}\left(q^{2}\right)=0$ and, from Eq. 4.9$), \hat{\Sigma}_{R, W W}^{(W Z)}\left(q^{2}\right)=\hat{\Sigma}_{R, Z Z}^{(W W)}\left(q^{2}\right)$.

\section{Appendix B}

In this Appendix, we give a brief discussion of the relations between the renormalization constants involved in the renormalization of the PT electroweak two-point functions. In particular, it is described how, as a result of the Ward identities obeyed by the PT $n$-point functions, the three effective charges and the effective weak mixing angle defined in Sec. 5 in terms of unrenormalized quantities take exactly the same form when written in terms of the corresponding renormalized quantities. Thus, they may be related directly to experimentally measureable quantities, as described in Sec. 7.

Renormalization constants are defined for the electromagnetic coupling and the gauge boson masses by

$$
\begin{aligned}
e^{2} & =Z_{e} e_{R}^{2}, \\
M_{Z}^{2} & =M_{R, Z}^{2}+\delta M_{Z}^{2}, \\
M_{W}^{2} & =M_{R, W}^{2}+\delta M_{W}^{2},
\end{aligned}
$$

and for the gauge fields by

$$
\begin{aligned}
\left(\begin{array}{c}
A \\
Z
\end{array}\right) & =\left(\begin{array}{cc}
Z_{A A}^{\frac{1}{2}} & Z_{A Z}^{\frac{1}{2}} \\
Z_{Z A}^{\frac{1}{2}} & Z_{Z Z}^{\frac{1}{2}}
\end{array}\right)\left(\begin{array}{c}
A_{R} \\
Z_{R}
\end{array}\right), \\
W^{ \pm} & =Z_{W}^{\frac{1}{2}} W_{R}^{ \pm} .
\end{aligned}
$$


The renormalized form $\hat{\Gamma}_{R, N C}$ of the matrix $\hat{\Gamma}_{N C}$ Eq. (5.1) collecting the PT bare neutral current two-point functions is then given by

$$
\hat{\Gamma}_{R, N C}=\left(\begin{array}{cc}
Z_{A A}^{\frac{1}{2}} & Z_{Z A}^{\frac{1}{2}} \\
Z_{A Z}^{\frac{1}{2}} & Z_{Z Z}^{\frac{1}{2}}
\end{array}\right) \hat{\Gamma}_{N C}\left(\begin{array}{cc}
Z_{A A}^{\frac{1}{2}} & Z_{A Z}^{\frac{1}{2}} \\
Z_{Z A}^{\frac{1}{2}} & Z_{Z Z}^{\frac{1}{2}}
\end{array}\right)
$$

Similarly, the renormalized form $\hat{\Gamma}_{R, C C}$ of the PT bare charged current two-point function is given by

$$
\hat{\Gamma}_{R, C C}=Z_{W}\left(q^{2}-M_{W}^{2}+\hat{\Sigma}_{W W}\right) .
$$

For the particular case of the on-shell scheme considered here, $\hat{\Gamma}_{R, N C}$ and $\hat{\Gamma}_{R, C C}$ take the form

$$
\begin{aligned}
\hat{\Gamma}_{R, N C} & =\left(\begin{array}{cc}
q^{2}+\hat{\Sigma}_{R, \gamma \gamma} & \hat{\Sigma}_{R, \gamma Z} \\
\hat{\Sigma}_{R, \gamma Z} & q^{2}-\bar{s}_{Z}+\hat{\Sigma}_{R, Z Z}
\end{array}\right), \\
\hat{\Gamma}_{R, C C} & =q^{2}-\bar{s}_{W}+\hat{\Sigma}_{R, W W}
\end{aligned}
$$

where $\bar{s}_{Z}$ and $\bar{s}_{W}$ are the $Z$ and $W$ complex pole positions, respectively.

It is convenient to introduce as auxilliary quantities the sine and cosine of the renormalized weak mixing angle, together with the corresponding renormalization constants:

$$
c_{\mathrm{w}}^{2} \equiv 1-s_{\mathrm{w}}^{2}=\frac{M_{W}^{2}}{N_{Z}^{2}}=\frac{M_{R, W}^{2}+\delta M_{W}^{2}}{M_{R, Z}^{2}+\delta M_{Z}^{2}}=Z_{c_{\mathrm{w}}} c_{R, \mathrm{w}}^{2} \equiv 1-Z_{s_{\mathrm{w}}} s_{R, \mathrm{w}}^{2} .
$$

Then, for example, in the on-shell scheme $c_{R, \mathrm{w}}^{2}=M_{R, W}^{2} / M_{R, Z}^{2}$, so that to one-loop order $Z_{c_{\mathrm{w}}}=1+\delta M_{W}^{2} / M_{W}^{2}-\delta M_{Z}^{2} / M_{Z}^{2}$.

The PT one-loop $n$-point functions have been shown explicitly to obey the same Ward identities as the corresponding tree level functions [11, 15]. The requirement that the renormalized functions satisfy the same identities as the unrenormalized functions then leads to relations among the renormalization constants. In particular, using these tree-level-like Ward identities, it is straightforward to obtain the following relations:

$$
\begin{aligned}
\left(\begin{array}{cc}
Z_{A A}^{\frac{1}{2}} & Z_{A Z}^{\frac{1}{2}} \\
Z_{Z A}^{\frac{1}{2}} & Z_{Z Z}^{\frac{1}{2}}
\end{array}\right) & =\frac{1}{Z_{e}^{\frac{1}{2}}}\left(\begin{array}{cc}
1 & \frac{s_{R, \mathrm{w}}}{c_{R, \mathrm{w}}}\left(Z_{s_{\mathrm{w}}}-1\right) \\
0 & Z_{S_{\mathrm{w}}}^{\frac{1}{2}} Z_{\mathrm{C}_{\mathrm{w}}}^{\frac{1}{2}}
\end{array}\right), \\
Z_{W}^{\frac{1}{2}} & =\frac{1}{Z_{e}^{\frac{1}{2}}} Z_{s_{\mathrm{w}}}^{\frac{1}{2}}
\end{aligned}
$$

Using the above definitions of the renormalized quantities $e_{R}, \Gamma_{R}, s_{R, \mathrm{w}}$ and $c_{R, \mathrm{w}}$, together with the above relations among the renormalization constants, the amplitude for the PT two-point component of the interaction between fermions with charges $Q, Q^{\prime}$ and isospins 
$I_{3}, I_{3}^{\prime}$ given in Eq. (5.2) in terms of bare quantities may be written

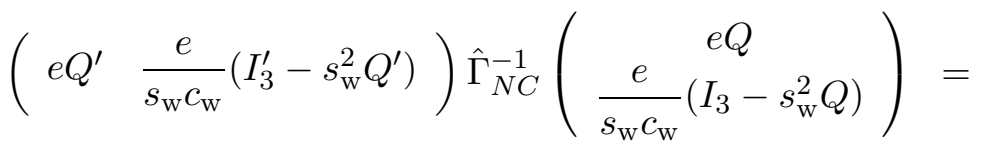

$$
\begin{aligned}
& \left(e_{R} Q^{\prime} \frac{e_{R}}{s_{R, \mathrm{w}} c_{R, \mathrm{w}}}\left(I_{3}^{\prime}-s_{R, \mathrm{w}}^{2} Q^{\prime}\right)\right) \hat{\Gamma}_{R, N C}^{-1}\left(\begin{array}{c}
e_{R} Q \\
\frac{e_{R}}{s_{R, \mathrm{w}} c_{R, \mathrm{w}}}\left(I_{3}-s_{R, \mathrm{w}}^{2} Q\right)
\end{array}\right) .
\end{aligned}
$$

Thus, the amplitude Eq. (5.2) takes exactly the same form when expressed in terms of the corresponding renormalized quantities. As a result, the products $e^{2} \hat{\Delta}_{\gamma}$ and $\left(e^{2} / s_{\mathrm{w}}^{2} c_{\mathrm{w}}^{2}\right) \hat{\Delta}_{Z}$ of couplings with the propagator functions Eqs. (5.3) and (5.4), and the effective weak mixing angle $s_{\mathrm{w}, \text { eff }}^{2}$ Eq. (5.5) defined from the diagonal form of the amplitude on the r.h.s. of Eq. (5.2), also take exactly the same form when expressed in terms of the renormalized quantities. An exactly similar result holds for the product $\left(e^{2} / s_{\mathrm{w}}^{2}\right) \hat{\Delta}_{W}$ in the PT Dyson-summed two-point component of the charge current interaction between fermions given in Eq. (5.6). Alternatively, multiplying out the components of Eqs. (B.3) and using the relations Eq. ( $\overline{B .8})$, one can verify the explicit relations

$$
\begin{aligned}
q^{2}+\hat{\Sigma}_{R, \gamma \gamma} & =\frac{1}{Z_{e}}\left(q^{2}+\hat{\Sigma}_{\gamma \gamma}\right), \\
q^{2}-\bar{s}_{Z}+\hat{\Sigma}_{R, Z Z}-\frac{\hat{\Sigma}_{R, \gamma Z}^{2}}{q^{2}+\hat{\Sigma}_{R, \gamma \gamma}} & =\frac{Z_{s_{\mathrm{w}}} Z_{c_{\mathrm{w}}}}{Z_{e}}\left(q^{2}-M_{Z}^{2}+\hat{\Sigma}_{Z Z}-\frac{\hat{\Sigma}_{\gamma Z}^{2}}{q^{2}+\hat{\Sigma}_{\gamma \gamma}}\right), \\
q^{2}-\bar{s}_{W}+\hat{\Sigma}_{R, W W} & =\frac{Z_{s_{\mathrm{w}}}}{Z_{e}}\left(q^{2}-M_{W}^{2}+\hat{\Sigma}_{W W}\right), \\
1+\frac{c_{R, \mathrm{w}}}{s_{R, \mathrm{w}}} \frac{\hat{\Sigma}_{R, \gamma Z}}{q^{2}+\hat{\Sigma}_{R, \gamma \gamma}} & =Z_{s_{\mathrm{w}}}\left(1+\frac{c_{\mathrm{w}}}{s_{\mathrm{w}}} \frac{\hat{\Sigma}_{\gamma Z}}{q^{2}+\hat{\Sigma}_{\gamma \gamma}}\right),
\end{aligned}
$$

among the combinations of self-energies appearing in $\hat{\Delta}_{\gamma}, \hat{\Delta}_{Z}, \hat{\Delta}_{W}$ and $s_{\mathrm{w} \text {,eff }}^{2}$ in Sec. 5 (for clarity, the above expressions have been given in terms of functions renormalized in the onshell scheme; they in fact hold for the components of $\Gamma_{R, N C}$ and $\Gamma_{R, C C}$ in any scheme).

The three effective charges $\alpha_{\text {eff }}, \alpha_{Z \text {,eff }}$ and $\alpha_{W \text {,eff }}$, obtained after factoring out the poles $\left(q^{2}\right)^{-1},\left(q^{2}-\bar{s}_{Z}\right)^{-1}$ and $\left(q^{2}-\bar{s}_{W}\right)^{-1}$ from the products of bare charges and diagonal bare propagator functions $e^{2} \hat{\Delta}_{\gamma},\left(e^{2} / s_{\mathrm{w}}^{2} c_{\mathrm{w}}^{2}\right) \hat{\Delta}_{Z}$ and $\left(e^{2} / s_{\mathrm{w}}^{2}\right) \hat{\Delta}_{W}$, respectively, and the effective weak mixing angle $s_{\mathrm{w}, \mathrm{eff}}^{2}$, defined from the diagonal bare neutral current amplitude, may therefore be expressed entirely in terms of the corresponding renormalized quantities, as in the second equality in each of Eqs. (5.11)-(5.13) and (5.5). 


\section{Appendix $\mathrm{C}$}

In this Appendix, we give the explicit expresssions for the polynomials $\tilde{F}_{i}(s, x), i=1,2 \ldots 5$, defined in Sec. 7 . Setting $D \equiv(1-\beta z)\left(1-z^{2}\right)$, they are as follows:

$$
\begin{aligned}
\tilde{F}_{1}(s, x)= & \frac{5}{4 D}\left\{\frac{3}{32}\left(16-(1-\beta z)\left(71-63 z^{2}\right)\right)-3(\beta+z) x\right. \\
& -\frac{21}{16}\left(16-(1-\beta z)\left(53-45 z^{2}\right)\right) x^{2}+7(\beta+z) x^{3} \\
& \left.+\frac{63}{32}\left(16-(1-\beta z)\left(43-35 z^{2}\right)\right) x^{4}\right\} \\
\tilde{F}_{2}(s, x)= & \frac{315}{128}\left\{3-30 x^{2}+35 x^{4}\right\} \\
\tilde{F}_{3}(s, x)= & \frac{5}{8}\left\{\frac{189}{8} z-21 x-\frac{945}{4} z x^{2}+35 x^{3}+\frac{2205}{8} z x^{4}\right\} \\
\tilde{F}_{4}(s, x)= & \frac{5}{4 D}\left\{-\frac{3}{2} z+3\left(1+z^{2}\right) x+21 z x^{2}-7\left(1+z^{2}\right) x^{3}-\frac{63}{2} z x^{4}\right\} \\
\tilde{F}_{5}(s, x)= & \frac{5}{8\left(1-z^{2}\right)}\left\{\frac{3}{16}\left(15-70 z^{2}+63 z^{4}\right)+3 z\left(5-7 z^{2}\right) x-\frac{21}{8}\left(5-42 z^{2}+45 z^{4}\right) x^{2}\right. \\
& \left.-7 z\left(3-5 z^{2}\right) x^{3}+\frac{63}{16}\left(3-30 z^{2}+35 z^{4}\right) x^{4}\right\}
\end{aligned}
$$

Note that $\tilde{F}_{2}(s, x)$ is proportional to the fourth Legendre polynomial $P_{4}(x)$.

\section{References}

[1] M. Gell-Mann and F.E. Low, Phys. Rev. 95 (1954) 1300.

[2] G. 't Hooft, in The Whys of Subnuclear Physics, ed. A. Zichichi (Plenum Press, New York, 1979); B. Lautrup, Phys. Lett. B69 (1977) 109; G. Parisi, Phys. Lett. B76 (1978) 65 and Nucl. Phys. B150 (1979) 163; F. David, Nucl. Phys. B234 (1984) 237 and Nucl. Phys. B263 (1986) 637; A.H. Mueller, Nucl. Phys. B250 (1985) 327 and Phys. Lett. B308 (1993) 355; V.I. Zakharov, Nucl. Phys. B385 (1992) 452; M. Beneke and V.I. Zakharov, Phys. Rev. Lett. 69 (1992) 2472; M. Beneke, Phys. Lett. B307 (1993) 154 and Nucl. Phys. B405 (1993) 424; M. Beneke and V.M. Braun, Nucl. Phys. B426 (1994) 301; D. Broadhurst, Z. Phys. C58 (1993) 339; M. Neubert and C.T. Sachrajda, Nucl. Phys. B438 (1995) 235; S. Peris and E. de Rafael, Phys. Lett. B387 (1996) 603.

[3] A. Pilaftsis, Z. Phys. C47 (1990) 95; A. Sirlin, Phys. Rev. Lett. 67 (1991) 2127 and Phys. Lett. B267 (1991) 240; U. Baur and D. Zeppenfeld, Phys. Rev. Lett. 75 (1995) 1002 and references therein. 
[4] J. Ellis, S. Kelley and D. Nanopoulos, Phys. Lett. B260 (1991) 131; U. Amaldi, W. de Boer and H. Furstenau, Phys. Lett. B260 (1991) 447; P. Langacker and N. Polonsky, Phys. Rev. D49 (1994) 1454 and references therein.

[5] N.J. Watson, in Proceedings of the Ringberg Workshop on Perspectives for Electroweak Interactions in $e^{+} e^{-}$Collisions, Ringberg, Germany, ed. B.A. Kniehl, (World Scientific, Singapore, 1995), hep-ph/9506276.

[6] J. Papavassiliou and A. Pilaftsis, Phys. Rev. Lett. 75 (1995) 3060 and Phys. Rev. D53 (1996) 2128.

[7] J. Papavassiliou and A. Pilaftsis, Phys. Rev. D54 (1996) 5315.

[8] N.J. Watson, Nucl. Phys. B494 (1997) 388.

[9] J.M. Cornwall, in Proceedings of the French-American Seminar on Theoretical Aspects of Quantum Chromodynamics, Marseille, France, 1981, ed. J.W. Dash (Centre de Physique Théorique report no. CPT-81/P-1345, 1982).

[10] J.M. Cornwall, Phys. Rev. D26 (1982) 1453.

[11] J.M. Cornwall and J.M. Papavassiliou, Phys. Rev. D40 (1989) 3474; J. Papavassiliou, Phys. Rev. D47 (1992) 4728;

[12] J. Papavassiliou, Phys. Rev. D41 (1990) 3179;

[13] G. Degrassi and A. Sirlin, Phys. Rev. D46 (1992) 3104.

[14] N.J. Watson, Phys. Lett. B349 (1995) 155.

[15] G. Degrassi, B. Kniehl and A. Sirlin, Phys. Rev. D48 (1993) 3963; J. Papavassiliou and K. Philippides, Phys. Rev. D48 (1993) 4255 and Phys. Rev. D52 (1995) 2355; J. Papavassiliou and C. Parrinello, Phys. Rev. D50 (1994) 3059; J. Papavassiliou, Phys. Rev. D50 (1994) 5958; J. Papavassiliou and A. Sirlin, Phys. Rev. D50 (1994) 5951; J. Papavassiliou, Phys. Rev. D51 (1995) 856; K. Sasaki, Phys. Lett. B369 (1996) 117 and Nucl. Phys. B472 (1996) 271.

[16] W. Alles, Ch. Boyer and A.J. Buras, Nucl. Phys. B119 (1977) 125.

[17] K. Fujikawa, B.W. Lee and A.I. Sanda, Phys. Rev. D6 (1972) 2923.

[18] W. Beenakker et al., The Fermion-Loop Scheme for Finite Width Effects in $e^{+} e^{-}$Annihilation into Four Fermions, hep-ph/9612260. 
[19] C. Itzykson and J.B. Zuber, Quantum Field Theory (McGraw-Hill, New York, 1980); L.S. Brown, Quantum Field Theory (Cambridge University Press, Cambridge, 1992); M. Veltman, Diagrammatica: The Path to Feynman Diagrams (Cambridge University Press, Cambridge, 1994); S. Weinberg, The Quantum Theory of Fields (Cambridge University Press, Cambridge, 1995).

[20] B.N. Taylor and E.R. Cohen, Recommended Values of the Fundamental Physical Constants: A Status Report, J. Res. Natl. Inst. Stand. Technol. 95 (1990) 497.

[21] G. 't Hooft, Nucl. Phys. B33 (1971) 173.

[22] C.H. Llewellyn Smith, Phys. Lett. B46 (1973) 233.

[23] J.M. Cornwall, D.N. Levin and G. Tiktopoulos, Phys. Rev. Lett. 30 (1973) 1268.

[24] W. Hollik, Electroweak Theory, lectures given at the 5th Hellenic School and Workshops on Elementary Particle Physics, 3-24 Sept. 1995, Corfu, Greece. hep-ph/9602380.

[25] G. Passarino and M. Veltman, Nucl. Phys. B160 (1979) 151; G. Degrassi and A. Sirlin, Nucl. Phys. B383 (1992) 73.

[26] L. Baulieu and R. Coquereaux, Ann. Phys. 140 (1982) 163; D.C. Kennedy and B.W. Lynn, Nucl. Phys. B322 (1989) 1; K. Phillippides and A. Sirlin, Phys. Lett. B367 (1996) 377.

[27] L.F. Abbott, Nucl. Phys. B185 (1981) 189, and references therein.

[28] A. Denner, G. Weiglein and S. Dittmaier, Phys. Lett. B333 (1994) 420 and Nucl. Phys. B440 (1995) 95.

[29] S. Hashimoto, J. Kodaira, Y. Yasui and K. Sasaki, Phys. Rev. D50 (1994) 7066.

[30] E. de Rafael and N.J. Watson, unpublished.

[31] A. Pilaftsis, Nucl. Phys. B487 (1997) 467.

[32] A. Denner, S. Dittmaier and G. Weiglein, in Proceedings of the 1994 Zeuthen Workshop on Physics at LEP200 and Beyond, Nucl. Phys. Proc. Suppl. B37 (1994) 87, hepph/9406400; in Proceedings of the Ringberg Workshop on Perspectives for Electroweak Interactions in $e^{+} e^{-}$Collisions, Ringberg, Germany, ed. B.A. Kniehl, (World Scientific, Singapore, 1995), hep-ph/9505271; lecture given at the XXXVI Cracow School of Theoretical Physics, Zakopane, Poland, June 1996, hep-ph/9609422.

[33] R.G. Stuart, Phys. Lett. B262 (1991) 113, Phys. Lett. B272 (1991) 353, Phys. Rev. Lett. 70 (1993) 3193 and in Proceedings of the Ringberg Workshop on Perspectives for 
Electroweak Interactions in $e^{+} e^{-}$Collisions, Ringberg, Germany, ed. B.A. Kniehl, (World Scientific, Singapore, 1995).

[34] M. Bilenky, J.L. Kneur, F. Renard and D. Schildknecht, Nucl. Phys. B409 (1993) 22.

[35] K. Hagiwara, D. Haidt, C.S. Kim and S. Matsumoto, Z. Phys. C64 (1994) 559. 